Draft VERSion MAY 24, 2022

Preprint typeset using $\mathrm{L}^{A} \mathrm{~T}_{\mathrm{E}} \mathrm{X}$ style emulateapj v. 11/10/09

\title{
FORMATION OF ORGANIC MOLECULES AND WATER IN WARM DISK ATMOSPHERES
}

\author{
JOAN R. NAJITA \\ National Optical Astronomy Observatory, 950 N. Cherry Avenue, Tucson, AZ, 85719
}

Máté ÁdÁmKovics And Alfred E. Glassgold

Astronomy Department, University of California, Berkeley, CA 94720

Draft version May 24, 2022

\begin{abstract}
Observations from Spitzer and ground-based infrared spectroscopy reveal significant diversity in the molecular emission from the inner few AU of $\mathrm{T}$ Tauri disks. We explore theoretically the possible origin of this diversity by expanding on our earlier thermal-chemical model of disk atmospheres. We consider how variations in grain settling, X-ray irradiation, accretion-related mechanical heating, and the oxygen-to-carbon ratio can affect the thermal and chemical properties of the atmosphere at $0.25-$ $40 \mathrm{AU}$. We find that these model parameters can account for many properties of the detected molecular emission. The column density of the warm (200-2000K) molecular atmosphere is sensitive to grain settling and the efficiency of accretion-related heating, which may account, at least in part, for the large range in molecular emission fluxes that have been observed. The dependence of the atmospheric properties on the model parameters may also help to explain trends that have been reported in the literature between molecular emission strength and mid-infrared color, stellar accretion rate, and disk mass. We discuss whether some of the differences between our model results and the observations (e.g., for water) indicate a role for vertical transport and freeze-out in the disk midplane. We also discuss how planetesimal formation in the outer disk (beyond the snowline) may imprint a chemical signature on the inner few $\mathrm{AU}$ of the disk and speculate on possible observational tracers of this process.
\end{abstract}

Subject headings: (stars:) circumstellar matter — (stars:) planetary systems: protoplanetary disks (stars:) planetary systems: formation

\section{INTRODUCTION}

Recent work with the Spitzer Space Telescope has found that emission from water and organic molecules is common in spectra of $\mathrm{T}$ Tauri stars (Carr \& Najita 2011, 2008; Salyk et al. 2011, 2008; Pontoppidan et al. 2010; Pascucci et al. 2009), with the emission likely arising from the inner few AU region of the circumstellar disk (Carr \& Najita 2008; Salyk et al. 2008). These new molecular diagnostics complement the more familiar near-infrared molecular diagnostics $(\mathrm{CO}$, water, $\mathrm{OH})$ that probe disk radii $\lesssim 1 \mathrm{AU}$ (see Najita et al. 2007; Carmona 2010 for recent reviews). Since T Tauri disks are optically thick in the continuum, these molecular emission features presumably arise from a temperature inversion region in a warm disk atmosphere. The possibility of warm molecular emission from the atmospheres of inner disks is supported by thermal-chemical models of disk atmospheres (Glassgold \& Najita 2001; Glassgold et al. 2004; Kamp \& Dullemond 2004; Willacy \& Woods 2009; Nomura \& Millar 2005; Gorti \& Hollenbach 2008).

One of the interesting properties of the mid-infrared molecular emission observed with Spitzer is that the fluxes and relative strengths of molecular features vary among the T Tauri stars (Carr \& Najita 2011; Salyk et al. 20011; Pontoppidan et al. 2010; Pascucci et al. 2009). The strength of the water emission varies by a factor of $>30$ (e.g., Pontoppidan et al. 2010), and an equally large range is found for the strength of the $\mathrm{HCN}$ emission (e.g., Salyk et al. 2011). The water emission can be brighter or fainter than the $\mathrm{HCN}$ emission, and
$\mathrm{HCN}$ is usually stronger than $\mathrm{C}_{2} \mathrm{H}_{2}$ among $\mathrm{T}$ Tauri stars but is sometimes weaker (Carr \& Najita 2011; Salyk et al. 2011; Pontoppidan et al. 2010; Pascucci et al. 2009). What is the origin of these variations in flux and relative strength?

Several interesting possibilities are suggested by trends in the demographics of molecular emission sources. While $\mathrm{HCN}$ is typically stronger than $\mathrm{C}_{2} \mathrm{H}_{2}$ in $\mathrm{T}$ Tauri stars, Pascucci et al. (2009) found $\mathrm{C}_{2} \mathrm{H}_{2}$ to be stronger than $\mathrm{HCN}$ in median spectra of brown dwarfs. Pascucci et al. suggested that this dichotomy arises because of the stronger UV field of T Tauri stars, which enhances the dissociation of $\mathrm{N}_{2}$ and the formation of $\mathrm{HCN}$ in those systems.

Further possible trends in HCN emission strength were noted by Teske et al. (2010). They found that the HCN emission strength of $\mathrm{T}$ Tauri stars appears to increase with stellar accretion rate and with X-ray luminosity, suggesting that these parameters may play a role in the molecular emission fluxes. They suggested that the trend of HCN flux with stellar accretion rate might result from enhanced accretion-related mechanical heating in the disk atmosphere, along the lines explored theoretically by Glassgold et al. (2009). These authors had previously suggested that increased rates of accretionrelated mechanical heating can increase the column density of warm water in the T Tauri disk atmosphere, producing stronger water emission. A similar effect might also be expected for other molecular species. Consistent with this perspective, Salyk et al. (2011) have reported 
a higher detection rate of molecular emission in systems with larger $\mathrm{H} \alpha$ equivalent widths (and tentatively also with stellar accretion rate), which they interpret as suggesting that accretional heating plays a role in enhancing the line emission. Salyk et al. also find that molecular emission is preferentially detected from disks that have experienced more grain settling, as indicated by their mid-infrared colors.

As an alternate explanation for their results, Teske et al. also discussed the possibility that increased UV irradiation produced at higher stellar accretion rates might enhance the HCN abundance, as discussed by Pascucci et al. (2009). Little interpretation was offered for the possible trend they found with $L_{\mathrm{X}}$, other than that Xray irradiation may help to synthesize HCN by raising the abundance of molecular ions and radicals.

Carr \& Najita (2011) also reported a possible relation between the $\mathrm{HCN} / \mathrm{H}_{2} \mathrm{O}$ emission flux ratio and disk mass in their small sample of $\mathrm{T}$ Tauri star spectra. The sources with the largest $\mathrm{HCN} / \mathrm{H}_{2} \mathrm{O}$ flux ratios are those with the largest disk masses. They note that such a trend might be expected as a consequence of planetesimal and protoplanet formation in the outer disk. As described by Ciesla \& Cuzzi (2006 and references therein), because icy bodies ranging from centimeter to kilometer and larger size migrate at different rates, their migration can lead to enhancements or depletions of water vapor in the disk that may vary as a function of both disk radius and time. Since water is a significant reservoir of oxygen, these effects can lower or raise the gas-phase oxygen-to-carbon $(\mathrm{O} / \mathrm{C})$ ratio in the inner disk and possibly affect the $\mathrm{HCN}$ and $\mathrm{H}_{2} \mathrm{O}$ abundances in the manner observed. More generally, because it is difficult to diagnose the formation of kilometer-sized planetesimals, the building blocks of planets, by other means, it is interesting to explore whether their formation may induce this (or any other) chemical signature in the inner disk that may signal their formation.

Here we explore the above ideas by expanding on our earlier thermal-chemical model of disk atmospheres. Models of disk atmospheres are in an early stage of development. In part, this is because disks are complex systems, and many processes potentially play a role in determining the thermal-chemical properties of their atmospheres. The relevant processes include those familiar from the study of molecular clouds and photodissociation regions (e.g., UV and X-ray irradiation and their roles in heating, photochemistry, and ionization; neutral and ion-molecule reactions; and the freeze out and desorption of molecules on grains). Other potentially important processes are more unique to circumstellar disks and are generally less well understood, both in terms of how they operate and their impact on the thermalchemical properties of disks. These include grain growth and settling, mechanical heating related to the magnetorotational instability, and radial and/or vertical transport of gas and solids. Models are developed to gain insight into how these processes affect disk atmospheres, with earlier studies having concentrated on different sets of processes and the interplay between them.

In our previous work, we constructed a model of inner disk atmospheres that includes the thermal decoupling of the gas and dust (e.g., Glassgold et al. 2001, 2004; see also Kamp \& Dullemond 2004; Nomura \& Millar 2005), was based on X-ray heating and ionization, and included effects such as grain growth and accretion-related mechanical heating (Glassgold et al. 2004). We used this model to study the gas-phase chemistry of water, including the effect of $\mathrm{H}_{2}$ formation on warm grain surfaces (Glassgold et al. 2009). Other studies have emphasized the effect of UV irradiation on the disk (e.g., Nomura \& Millar 2005; Woitke et al. 2009; Woods \& Willacy 2009; Bethell \& Bergin 2009), included freeze-out and desorption in the disk midplane or outer disk (e.g., Markwick et al. 2002; Woods \& Willacy 2009; Walsh et al. 2010), and explored the effect of transport processes on disk chemistry (e.g., Ilgner et al. 2004, Semenov et al. 2006; Willacy et al. 2006; Heinzeller et al. 2011), processes that we have yet to include. In this paper, we build on our earlier work, implementing an improved X-ray ionization theory and an expanded chemical network and explore how variations in X-ray irradiation, accretionrelated mechanical heating, and the $\mathrm{O} / \mathrm{C}$ ratio affect the thermal and chemical properties of the atmosphere.

Our study complements other recent work on the chemistry of inner disks (e.g., Agúndez et al. 2008; Woitke et al. 2009; Willacy \& Woods 2009; Nomura et al. 2009; Kress et al. 2010; Markwick et al. 2002). The work of Agúndez et al. (2008) is perhaps the closest in spirit to our own study. In their study, they addressed the steady-state properties of the disk atmosphere, assuming that the disk is strongly irradiated by FUV photons and adopting an extended neutral chemistry network that includes carbon species. Our study complements the Agúndez et al. study in that we include $\mathrm{X}$ ray (but not UV) irradiation of the disk. While a more complete model would include UV irradiation, here we explore how well a model that excludes UV can do in accounting for the existing observations.

\section{DESCRIPTION OF THE MODEL}

We study the hydrocarbon chemistry of protoplanetary disks with the steady-state thermal-chemical model developed by Glassgold, Najita \& Igea (2004; henceforth GNI04). This model is based on the D'Alessio et al. (1999) disk atmosphere for a generic T Tauri star of mass $0.5 M_{*}$, blackbody temperature $T_{*}=4000 \mathrm{~K}$, and accretion rate $10^{-8} M_{\odot} \mathrm{yr}^{-1}$. The disk is irradiated by stellar X-rays (which affect the temperature, ionization, and chemical structure of the gas), but not by far ultraviolet (FUV) radiation. GNI04 used the dust temperature obtained by D'Alessio et al. and calculated the gas temperature on the basis of heating by collisions with dust grains, X-rays, and accretion-related mechanical heating, balanced by a variety of line cooling processes and gas-dust collisions. As a result, the gas temperature is elevated over the dust temperature over much of the atmosphere.

We adopt a steady state treatment of the chemical kinetics rather than a time-dependent one. This approach is supported in part by the warm gas temperatures inferred for the Spitzer molecular emission (300-1000K), which generally imply short chemical timescales compared to transport timescales. The chemistry in GNI04 was limited to 25 species for the elements $\mathrm{H}, \mathrm{He}, \mathrm{C}$ and $\mathrm{O}$, and it contained 115 reactions. In a subsequent study of the water chemistry of protoplanetary disks, Glassgold, Meijerink \& Najita (2009; henceforth GMN09) corrected 
and expanded the chemistry to $\sim 100$ species and $\sim 400$ reactions.

GMN09 emphasized the role of $\mathrm{H}_{2}$ formation on warm dust grains, following the theory of Cazaux \& Tielens (2002, 2004; Cazaux et al. 2005) who showed that $\mathrm{H}_{2}$ can form with moderate efficiency $(\lesssim 0.2)$ on warm dust with temperatures up to $\sim 900 \mathrm{~K}$. Other recent studies of disk atmospheres have also employed this process in their models (e.g., Heinzeller et al. 2011; Woitke et al. 2009; Willacy \& Woods 2009; and Woods \& Willacy 2009). Enhanced $\mathrm{H}_{2}$ formation on grains appears to play an important role in producing significant warm molecular columns in models such as ours that include grain growth (Woods \& Willacy 2009). If grain growth is not included, enhanced $\mathrm{H}_{2}$ formation on grains can lead to the overproduction of warm molecular emission (e.g., Heinzeller et al. 2011).

In this paper we build on our previous models by employing an expanded chemical network that includes hydrocarbon, nitrogen and sulfur chemistry. The current model includes an enhanced theory of X-ray ionization that treats individually the nine most abundant heavy atoms (Adamkovics, Glassgold, \& Meijerink 2011; henceforth AGM11) in addition to $\mathrm{C}, \mathrm{N}$ and $\mathrm{O}$. The chemical network now contains $\sim 115$ species and $\sim 1200$ reactions. Even so, the molecular complexity is limited to molecules (and ions) of 6 atoms or less, with hydrocarbons containing no more than two carbon atoms, as in $\mathrm{C}_{2} \mathrm{H}_{4}$

The reaction list contains neutral and ion-molecule reactions, X-ray interactions, dissociative and radiative recombination, and radiative association. We evaluated the reactions in our model using the primary literature and the critical evaluations of Baulch et al. (2005) for neutral reactions and Anicich (1993) for ion-molecule reactions. We have also consulted the NIST Chemical Kinetics Database (http://kinetics.nist.gov) as well as the UMIST (Woodall et al. 2007) and KIDA (Wakelam, 2009) collections.

Irradiation by stellar and/or interstellar FUV is not included in the present model, an assumption that would apply when the disk is well shielded from UV (e.g., by an intervening funnel flow). This assumption may also be more generally valid in the context of heating. Earlier work (e.g., Nomura et al. 2007) has shown that UV heating due to the photoelectric effect is significantly reduced relative to other heating processes when grains have grown and settled to the extent that has been deduced for T Tauri disks (Furlan et al. 2006). This, and the uncertain PAH abundance of T Tauri disks, mitigates our neglect of UV heating, a topic that we return to in section 4. More seriously, UV photodissociation likely affects the molecular abundances as we also discuss in section 4.

One obvious difference between our X-ray irradiated model and others that focus on UV irradiation is that in our model molecules are destroyed by $\mathrm{H}^{+}$and $\mathrm{He}^{+}$. In the presence of UV irradiation, photodissociation would also play a significant role in destroying molecules. In a similar vein, UV irradiation will also alter the ionization and temperature structure from that considered here. Another difference between UV and X-ray irradiated models is that $\mathrm{X}$-rays are also able to ionize species that are unavailable to UV photons. For example, al- though FUV photons are unable to directly ionize $\mathrm{N}$ and $\mathrm{N}_{2}$, ion-neutral reactions of $\mathrm{N}_{2}$ with $\mathrm{H}_{3}^{+}$and $\mathrm{He}^{+}$(which are generated by $\mathrm{X}$-rays) can activate the nitrogen chemistry.

We include the formation of $\mathrm{H}_{2}$ on dust grains in the same way as GMN09 (their eqs. 14 and 15), following the theory of Cazaux \& Tielens. At the suggestion of Bai \& Goodman (2009), Cazaux \& Tielens (2010) have since corrected their calculations, but the changes are only of a quantitative nature. We ignore the possibility of forming other molecules on grains. GMN09 found that ignoring $\mathrm{H}_{2} \mathrm{O}$ formation on grains did not much affect the distribution of gaseous $\mathrm{H}_{2} \mathrm{O}$. We also ignore cooling by $\mathrm{H}_{2} \mathrm{O}$ rotational transitions as well as heating due to UV absorption by $\mathrm{H}_{2} \mathrm{O}$. Water line emission has been measured to be a significant coolant for disk atmospheres (Pontoppidan et al. 2010). These limitations will be remedied in future reports.

As discussed in more detail in AGM11, the abundances of the heavy elements play an important role in the chemistry of the disk atmosphere, because they are the dominant charge carriers below the atomic to molecular transition. Measurements are not available for the absolute gas-phase abundances of the elements in thick molecular clouds, not to mention protoplanetary disks. The only abundance determinations come from UV absorption line spectroscopy of relatively diffuse interstellar clouds $\left(A_{V} \lesssim 2 \mathrm{mag}\right.$ ) obtained from observations made with satellite observatories. The measured abundances of the heavy elements vary from cloud to cloud, and we continue to use those abundances reported for the very well studied cloud towards $\zeta$ Oph (Savage \& Sembach 1996); as shown in Table 1. The last column shows the depletion factors, defined as the ratio of solar to interstellar abundances, with the former coming from Table 1 of the review of solar abundances by Asplund et al. (2009). As discussed in this paper and in Jenkin's (2009) review of interstellar abundances, both sets of values have significant uncertainties, in some cases factors of a few. Jenkins highlights the case of S, where the UV observations suggest essentially no depletion in interstellar clouds, despite the fact that this element is seen in meteorites.

Although we use the depleted interstellar abundances in Table 1 for the preliminary modeling purposes of this paper, we recognize that the abundances of the heavy elements in diffuse interstellar clouds may well have been altered in dense clouds and by the formation and evolution of protoplanetary disks. The determination of relative abundances in disks remains a primary goal of observations of the emission line fluxes of their atoms and molecules. As discussed in Section 1, we will examine one particular modification of the elemental abundances, the variation of the oxygen-to-carbon ratio $(\mathrm{O} / \mathrm{C})$ that may be induced by the adsorption of water on solid bodies and the transport of those bodies between large and small radii.

The molecular abundances in the inner disk atmosphere are affected by the level of X-ray irradiation, the strength of the accretion-related mechanical heating, and the oxygen-to-carbon ratio $(\mathrm{O} / \mathrm{C})$. Of course they also change with the radial distance from the star. We have investigated these effects by carrying out variational calculations about a "reference case" defined in Table 2. The X-ray variations are parameterized by the X-ray 
luminosity $L_{\mathrm{X}}$ and the $\mathrm{X}$-ray temperature $T_{\mathrm{X}}$, and the mechanical heating by the parameter $\alpha_{h}$ in Eq. (12) in GNI04,

$$
\Gamma_{\text {acc }}=\frac{9}{4} \alpha_{h} \rho c^{2} \Omega,
$$

where $\rho$ is the local mass density, $c$ is the isothermal sound speed, $\Omega$ is the angular rotation speed and $\alpha_{h}$ is a phenomenological parameter specifying the strength of the heating. As discussed by GMN09 in connection with their Equation 1, the grain surface area per unit volume is proportional to the dust-to-gas ratio of the disk, $\rho_{\text {dust }} / \rho$, and the inverse of the geometric mean $\left(a_{g}\right)$ of the minimum and the maximum grain radii in an MRN dust size distribution $\left(n(a) \propto a^{-3.5}\right)$.

Table 2 gives the range of parameter variations we considered. As in our previous papers, the dust-to-gas ratio was fixed at 0.01 , and we considered values of $a_{q}$ that are 32 and 320 times larger than the usual interstellar value of $0.0224 \mu \mathrm{m}$, i.e., 0.707 and $7.07 \mu \mathrm{m}$. This range is consistent with the dust depletion factors $(\sim 100-1000)$ that are found for the upper disk layers of $\mathrm{T}$ Tauri stars based on their mid-infrared colors (Furlan et al. 2006). We adopted $L_{\mathrm{X}}$ and $T_{\mathrm{X}}$ values that are typical of T Tauri stars $\left(L_{X}=10^{29}-10^{31} \mathrm{erg} \mathrm{s}^{-1}\right.$; Feigelson et al. 2005) as well as lower values of $L_{\mathrm{X}}$ down to $10^{26} \mathrm{erg} \mathrm{s}^{-1}$ in order to illustrate the role of the X-rays.

The parameter $\alpha_{h}$ was varied so that accretion heating was either non-dominant $\left(\alpha_{h}=0.01\right)$ or dominant $\left(\alpha_{h}=1\right)$ as a heat source. The lower value is motivated by the viscosity parameter for $\mathrm{T}$ Tauri disks, which has been estimated as $\alpha \simeq 0.01$ as a vertical average in a steady state disk (e.g., Hartmann et al. 1998). The larger value is motivated by $3 \mathrm{D}$ MHD calculations that suggest that turbulent accretion disks driven by the magnetorotational instability experience greater energy dissipation at the disk surface than in a classical " $\alpha$-disk". A recent calculation by Hirose \& Turner (2011) suggests that the energy dissipation rate rises toward the disk surface, reaching values that correspond to $\alpha_{h} \simeq 1$.

The range of radii we considered covers the inner disk region probed by Spitzer (the inner few AU) and the larger radii relevant to Herschel (out to $40 \mathrm{AU}$ ). We considered a range in the $\mathrm{O} / \mathrm{C}$ ratio in order to explore the dehydrated to super-hydrated regimes described in Ciesla \& Cuzzi (2006). Those models indicate that the (vertically averaged) water vapor concentration can increase by up to an order of magnitude (e.g., their Figure 1), or decrease by more than an order of magnitude (e.g., their Figure 3) in the inner few AU region. We translated this into an enhancement or decrement in the oxygen abundance by up to an order of magnitude relative to the standard case. We describe the results of our model calculations for the reference case in Section 3.1 and the results for the variations in Section 3.2.

\section{RESULTS}

\subsection{Reference Case}

Figure 1 shows the vertical variation of some of the basic physical quantities of the disk atmosphere for a range of radial distances in the inner disk between $0.25 \mathrm{AU}$ and $20 \mathrm{AU}$. In all three panels, the abscissa is the vertical column density $N_{\mathrm{H}}$, integrated downward into the disk from above. The top panel shows the ionization rate mul- tiplied by the inverse-square dilution factor. The similarity of the profiles at the different radii shown illustrates the usefulness of characterizing disk properties as a function of vertical column. Note that Compton scattering, which is not treated here, begins to become important for column densities approaching the maximum value in the figure $N_{\mathrm{H}}=10^{24} \mathrm{~cm}^{-2}$.

The temperature variation with column density (Fig. 1; middle panel) shows a particularly rapid drop at vertical column densities $N_{\mathrm{H}} \sim 0.6-1.5 \times 10^{21} \mathrm{~cm}^{-2}$. The temperature is high, in the range $4000-5000 \mathrm{~K}$, at column densities below this value, but it then drops into the warm 200-2000 K range at larger column densities. The atmosphere remains warm until collisions with dust grains bring the gas temperature down to the dust temperature; this occurs somewhere in the column density range $N_{\mathrm{H}}=10^{22}-10^{23} \mathrm{~cm}^{-2}$, with the value depending on radius. The $200-2000 \mathrm{~K}$ region (henceforth the "warm atmosphere") is a region of high density with $n_{\mathrm{H}}=3 \times 10^{9}-3 \times 10^{10} \mathrm{~cm}^{-3}$ in the reference case. As shown in the bottom panel of Fig. 1, the ionization fraction decreases by factors of $\sim 100$ where the gas temperature drops abruptly and the transition from $\mathrm{H}$ to $\mathrm{H}_{2}$ occurs.

Many molecular abundances also increase by large amounts as the temperature and ionization fraction drop, led by the atomic to molecular hydrogen transition shown in the bottom panel of Figure 1. The change from a primarily atomic to a primarily molecular medium, and from higher to lower levels of temperature and ionization, are the manifestations of a thermal-chemical transition. This transition is mediated thermally by the onset of strong molecular line cooling that causes the sharp decrease in temperature. The chemical transition to molecules results from the decrease in the ionization level of $\mathrm{H}^{+}$and $\mathrm{He}^{+}$, which destroy molecules, and the suppression of chemical temperature-dependent reactions that destroy them.

Thermal-chemical transitions are commonly found among models in the literature, although the details of the transition may differ depending on the irradiation field and other model properties. The sharpness of the transition is one property that can vary depending on the model parameters, and sharp transitions like that of the (X-ray irradiated) reference model may appear in UV irradiated models as well. The UV irradiated model of Woitke et al. (2009) is a good example. The electron fraction in that model varies sharply, from $10^{-4}$ to $10^{-5}$ at $z / r=0.15$ over a radial range from $\sim 0.5$ to $10 \mathrm{AU}$. The specific details differ from our model, although the possibility of sharp transitions is a common element. Models that include both UV and X-rays also find results similar to ours, e.g., a recent calculation by Woitke (2011, personal communication) has a similar temperature structure. Nomura et al. (2007) also find a sharp temperature decline when X-rays and UV are considered and grains have grown to large size. There is also diversity among Xray irradiated models: we find sharper transitions when mechanical heating is included, and shallower transitions when mechanical heating is weak (Glassgold et al. 2004, 2009; see also the following section).

The top two panels of Figure 2 show the transitions of carbon, oxygen and nitrogen to molecules for the ref- 
erence model at radii $0.25,1.0$, and $2.0 \mathrm{AU}$ (Figure 2a), and 4.0, 10, and $20 \mathrm{AU}$ (Figure 2b). The abundances in this figure are defined relative to that of all hydrogen nuclei, and the region below the main thermal-chemical transition near $N_{\mathrm{H}} \sim 1-3 \times 10^{21} \mathrm{~cm}^{-2}$ is emphasized. Focusing for the moment on the results for $r=1 \mathrm{AU}$, we find that, like the transition from atomic $\mathrm{H}$ to $\mathrm{H}_{2}$, the transition from atomic carbon to $\mathrm{CO}$ is complete, and $\mathrm{CO}$ remains abundant down to the largest vertical columns. In contrast, the conversion of all of the residual oxygen (that is not in $\mathrm{CO}$ ) into $\mathrm{H}_{2} \mathrm{O}$ occurs in the warm atmosphere $\left(N_{\mathrm{H}}=1-3 \times 10^{21} \mathrm{~cm}^{-2}\right)$, but the $\mathrm{H}_{2} \mathrm{O}$ abundance declines at larger columns where the gas is no longer warm, with $\mathrm{O}_{2}$ and $\mathrm{SO}_{2}$ as well as atomic $\mathrm{O}$ becoming the major reservoirs of the residual oxygen.

Several other recent thermal-chemical calculations also study $\mathrm{H}_{2} \mathrm{O}$ synthesis in disk atmospheres. In the model of Woitke et al. (2009), water is present in the disk atmosphere at $1 \mathrm{AU}$ although at much lower column densities $\left(<10^{14} \mathrm{~cm}^{-2}\right.$ above $\left.200 \mathrm{~K}\right)$ than found here. In the calculation of Willacy \& Woods (2009), a much larger column of warm water $\left(\sim 10^{19} \mathrm{~cm}^{-2}\right)$ is present and at higher temperature $(\gtrsim 1000 \mathrm{~K})$.

As shown in the second panel (from the top) of Figure 2 , the transformation from atomic to molecular nitrogen becomes complete in the warm zone, and atomic N remains fairly abundant throughout much of the inner disk. The bottom panel shows the molecular ions generated by X-ray ionization. Although they are important for the chemistry, they do not dominate the ionization balance, which is accomplished in this model by heavy atomic ions such as $\mathrm{Si}^{+}, \mathrm{Fe}^{+}$and $\mathrm{Mg}^{+}$. However, this conclusion is sensitive to the assumed elemental abundances, as discussed by AGM11.

We find that a thermal-chemical transition from hot and atomic to warm and molecular always occurs in Xray irradiated disks, at least out to $40 \mathrm{AU}$, although the details of the transition (such its location, sharpness, and other properties) are sensitive to the input parameters of the chemical and thermal model. We discuss this topic further in Section 3.2, where we describe variations about the reference model. In this section we focus on the molecular abundances found in the reference case.

The physical and chemical properties of the disk atmosphere that are reported here are consistent with those from our previous models (e.g., GNI04, GMN09). The present work does have some quantitative differences with our earlier work. These are associated with the many improvements in the physical-chemical model and the computational program employed here.

It is no accident that the transition to from atomic $\mathrm{C}$ to $\mathrm{CO}$ follows closely the temperature and ionization transitions in Figure 1, since $\mathrm{CO}$ is a dominant coolant in the thermal-chemical transition. The attainment of maximal $\mathrm{CO}$ abundance occurs relatively high in the disk atmosphere through the action of warm neutral radical reactions, as discussed in GNI04 (Sec. 3) and GMN09 (Sec. 2.2). The formation of the $\mathrm{OH}$ radical is a key step in the synthesis of $\mathrm{CO}$ and $\mathrm{H}_{2}$, through the endothermic reaction,

$$
\mathrm{O}+\mathrm{H}_{2} \rightarrow \mathrm{H}+\mathrm{OH}
$$

the rate coefficient recommended by Baulch et al. (1992) is

$$
k=8.5 \times 10^{-20} T^{2.67} e^{-3163 / T} \mathrm{~cm}^{3} \mathrm{~s}^{-1} .
$$

Water is then formed by an exothermic reaction with a modest barrier,

$$
\mathrm{OH}+\mathrm{H}_{2} \rightarrow \mathrm{H}_{2} \mathrm{O}+\mathrm{H} ;
$$

its rate coefficient is

$$
k=8.5 \times 1.70 \times 10^{-16} T^{1.60} e^{-1660 / T} \mathrm{~cm}^{3} \mathrm{~s}^{-1} .
$$

By contrast, $\mathrm{CO}$ is formed from $\mathrm{OH}$ by a fast neutral reaction without a significant barrier. Despite the barriers in Eqs. 3 and 5, water and $\mathrm{CO}$ are formed efficiently by the above reactions at temperatures as low as $\sim 250 \mathrm{~K}$.

This situation is not duplicated in the case of hydride formation for the two other abundant and chemically interesting heavy atoms, $\mathrm{C}$ and $\mathrm{N}$. In reactions analogous to Eq. 3, with $\mathrm{O}$ replaced by $\mathrm{C}$ and $\mathrm{N}$, the thermal barriers are now $\sim 12000 \mathrm{~K}$ instead of $\sim 3000 \mathrm{~K}$. These barriers suppress the formation of $\mathrm{CH}$ and $\mathrm{NH}$ by reactions with $\mathrm{H}_{2}$, and the inverse reactions, in which atomic $\mathrm{H}$ destroys $\mathrm{CH}$ and $\mathrm{NH}$, are both fast. In our X-ray irradiated models, the abundance of $\mathrm{H}$ decreases slowly below the thermal-chemical transition, so both suppressed formation (via reactions with $\mathrm{H}_{2}$ ) and rapid destruction (by $\mathrm{H}$; i.e., reactions analogous to those in Eqs. 1 and 3) imply that neutral production of carbon and nitrogen hydrides along the lines of oxygen-hydride synthesis is inefficient.

Nonetheless, the warm neutral chemistry that is responsible for most carbon and oxygen going into $\mathrm{CO}$ and $\mathrm{H}_{2} \mathrm{O}$ plays an important role in the synthesis of other molecules. This is shown in Figure 3, which is a highly schematic chemical network that shows how fast neutral reactions of $\mathrm{OH}$ with $\mathrm{N}, \mathrm{O}$ and $\mathrm{CO}$ generate other molecules such as $\mathrm{CO}_{2}, \mathrm{~N}_{2}$, and $\mathrm{SO}_{2}$ (not shown) and more complex molecules that will be discussed below. Thus the $\mathrm{OH}$ radical is the centerpiece of the warm chemistry of protoplanetary disk atmospheres.

As shown in Figure 3, the production of NO by the fast reaction,

$$
\mathrm{OH}+\mathrm{N} \rightarrow \mathrm{NO}+\mathrm{H} \quad k \sim 5 \times 10^{-11} \mathrm{~cm}^{3} \mathrm{~s}^{-1},
$$

leads to the formation of $\mathrm{N}_{2}$

$$
\mathrm{NO}+\mathrm{N} \rightarrow \mathrm{N}_{2}+\mathrm{O} \quad k \sim 4 \times 10^{-11} \mathrm{~cm}^{3} \mathrm{~s}^{-1} .
$$

The nitrogen hydrides can be synthesized by successive hydrogenation of the $\mathrm{N}^{+}$ion, produced by X-ray ionization, via successive reactions with $\mathrm{H}_{2}$, followed by dissociative recombination of the hydride ions. Ionhydrogenation becomes weak beyond $\mathrm{NH}_{3}{ }^{+}$, but proton transfer from molecular ions to neutral hydrides will produce hydride ions up to $\mathrm{NH}_{4}^{+}$. Thus a combination of ion-molecule and neutral reactions initiated by X-ray ionization can synthesize ammonia. A related sequence of neutral reactions starting with NO leads to the synthesis of $\mathrm{HCN}$,

$$
\begin{gathered}
\mathrm{NO}+\mathrm{C} \rightarrow \mathrm{CN}+\mathrm{O} \quad k \sim 3 \times 10^{-11} \mathrm{~cm}^{3} \mathrm{~s}^{-1}, \\
\mathrm{CN}+\mathrm{H}_{2} \rightarrow \mathrm{HCN}+\mathrm{H} \quad k(300 \mathrm{~K}) \sim 2.0 \times 10^{-14} \mathrm{~cm}^{3} \mathrm{~s}^{-1} .
\end{gathered}
$$

Although many reactions have been omitted from this brief description of the nitrogen chemistry for purposes of clarity, the middle panel of Fig. 2 shows that the main 
$\mathrm{C} \rightarrow \mathrm{CO}$ and $\mathrm{O} \rightarrow \mathrm{H}_{2} \mathrm{O}$ transitions are accompanied by the almost full conversion of $\mathrm{N}$ to $\mathrm{N}_{2}$. The transformation from $\mathrm{N}$ to $\mathrm{N}_{2}$ is mediated by neutral reactions as indicated in Fig. 3. It is accompanied by significant warm columns of other nitrogen-bearing molecules, such as $\mathrm{NO}, \mathrm{NH}_{3}, \mathrm{HCN}$ and $\mathrm{N}_{2} \mathrm{H}^{+}$.

The integrated treatment of both neutral and ionmolecule reactions is a feature of the underlying chemical model, with some molecular species demonstrating a greater sensitivity to X-ray irradiation than other species. The X-rays play a critical role in the synthesis of $\mathrm{NH}_{3}$ and $\mathrm{N}_{2} \mathrm{H}^{+}$. Since FUV radiation is unable to ionize $\mathrm{N}$ and $\mathrm{N}_{2}$ (which have ionization potentials of 14.5 and $15.6 \mathrm{eV}$, respectively), the X-rays are important in activating the nitrogen chemistry.

The hydrocarbon chemistry proceeds similarly to the nitrogen chemistry. The number of hydrogen atoms in a hydrocarbon molecule or ion can be increased by reactions with $\mathrm{H}_{2}$ or decreased by reactions with $\mathrm{H}$. Neutral hydrocarbons can be ionized by proton transfer from abundant molecular ions, such as $\mathrm{H}_{3}^{+}, \mathrm{HCO}^{+}$and $\mathrm{H}_{3} \mathrm{O}^{+}$, and neutralized by dissociative recombination with electrons; their charge is also affected by charge transfer processes. These types of reactions are believed to be operative for the hydrocarbon families of interest, those with one or two carbon atoms. A key point is that doublecarbon molecules are synthesized from those with just one carbon atom by insertion reactions with $\mathrm{C}$ and $\mathrm{C}^{+}$, as illustrated by the simplest examples,

$$
\begin{gathered}
\mathrm{C}+\mathrm{CH}_{n} \rightarrow \mathrm{C}_{2} \mathrm{H}_{n-1}+\mathrm{H} \quad(n=1-4), \\
\mathrm{C}^{+}+\mathrm{CH}_{n} \rightarrow \mathrm{C}_{2} \mathrm{H}_{n-1}^{+}+\mathrm{H} \quad(n=1-4),
\end{gathered}
$$

or by molecular ions as suggested by Woodall et al. (2007)

$$
\mathrm{CH}_{3}^{+}+\mathrm{C} \rightarrow \mathrm{C}_{2} \mathrm{H}^{+}+\mathrm{H}_{2} \text { and } \mathrm{CH}_{2}^{+}+\mathrm{C} \rightarrow \mathrm{C}_{2} \mathrm{H}^{+}+\mathrm{H} \text {. }
$$

These highly simplified remarks about hydrocarbon chemistry are closely aligned with the general discussion of Agúndez, Cernicharo \& Goicoechea (2008). In their model variation that is closest to our model, these authors use a photodissociation code to calculate the chemical structure of the surface region of the D'Alessio et al. (1999) disk model. They assume that the gas and dust temperatures are the same and that the dust is interstellar, and they consider stellar as well as interstellar UV radiation but ignore X-rays. Despite the many differences with their model, we draw the same general conclusion: hydrocarbons are predicted to be present in the top layers of protoplanetary disk atmospheres. There are of course quantitative differences between the results of Agúndez et al. and those reported here. We emphasize warm molecular column densities that arise in regions with $T \geq 200-300 \mathrm{~K}$, because they are related to measured fluxes. Agúndez et al. do not calculate gas temperatures, and they report total vertical column densities in the disk surface layer, almost all of which is cool gas $(<300 \mathrm{~K})$ at $1 \mathrm{AU}$.

Willacy \& Woods (2009) also synthesize a signficant column of warm $\mathrm{HCN}$. At $1 \mathrm{AU}$ they find a column of $\sim 4 \times 10^{16} \mathrm{~cm}^{-2}$ at a temperature $\gtrsim 1000 \mathrm{~K}$. As they note, the much lower columns of $\mathrm{C}_{2} \mathrm{H}_{2}$ that result in their models $\left(\sim 9 \times 10^{8} \mathrm{~cm}^{-2}\right)$ are likely due to the omission of reactions involving $\mathrm{H}_{2}$ with $\mathrm{C}_{2}$ or $\mathrm{C}_{2} \mathrm{H}$.

Despite the fact that $\mathrm{CO}$ tends to take up most of the available carbon, the hydrocarbon chemistry is still active after the main transition. This is a characteristic feature of the X-ray chemistry, where X-ray generated $\mathrm{He}^{+}$ions produce $\mathrm{C}^{+}$ions when they destroy $\mathrm{CO}$ by the ion-molecule reaction,

$$
\mathrm{He}^{+}+\mathrm{CO} \rightarrow \mathrm{C}^{+}+\mathrm{O}+\mathrm{He}
$$

Similar reactions, in which $\mathrm{He}^{+}$generates atomic ions from other molecules, are in fact quite common, notably for $\mathrm{N}_{2}$ and $\mathrm{CO}_{2}$. In the latter case $\mathrm{O}^{+}$is also produced.

In general, we find that both neutral and ion-molecule reactions contribute to the production and destruction of molecular species, with the relative importance of the two kinds of reactions varying with position in the disk as well as with species. Neutral reactions are more important in the warm disk surface, while ion-molecule reactions play a significant role in the cooler regions below. The species shown in Fig. 2 offer many challenges for the detection of molecules in the inner parts of protoplanetary disks, and some have already been observed, as discussed in Sec. 1.

We briefly summarize the results for the reference model at 1 AU, describing our results for the more abundant molecules. To do this, we take advantage of the monotonically decreasing run of temperature with increasing vertical column density (Fig. 1) and integrate the species' volumetric densities downward from the maximum $(2000 \mathrm{~K})$ to minimum $(200 \mathrm{~K})$ temperature of the warm atmosphere. This corresponds to the region just below the main thermal-chemical transition discussed in Section 2 and roughly to the region that can produce observable line emission in disks seen partly face-on. We often refer henceforth to these integrated columns simply as "warm columns".

- $\mathrm{H}_{2} \mathrm{O}$ forms in the same region as $\mathrm{CO}$ and reaches a comparable abundance, $\sim 10^{-4}$. The water abundance declines below $4 \times 10^{21} \mathrm{~cm}^{-2}$ before recovering its high abundance approaching $10^{24} \mathrm{~cm}^{-2}$. The resulting column density of warm water is $\sim 5 \times 10^{17} \mathrm{~cm}^{-2}$. In the region of reduced water abundances, several other species take up the available oxygen. Together with water they (i.e., $\mathrm{O}_{2}$ and $\mathrm{SO}_{2}$ ) would enhance the cooling above that provided by $\mathrm{O}$ and $\mathrm{CO}$, the two species that are contribute to the cooling in the present calculation.

- HCN is less abundant, reaching a maximum abundance of $\sim 10^{-7}$ in the warm atmosphere. The total column of warm $(>200 \mathrm{~K}) \mathrm{HCN}$ is $\sim 10^{15} \mathrm{~cm}^{-2}$.

- $\mathrm{C}_{2} \mathrm{H}_{2}$ is less abundant than $\mathrm{HCN}$, reaching a maximum abundance of $10^{-8}$ in the warm atmosphere. The column of warm $\mathrm{C}_{2} \mathrm{H}_{2}$ is $\sim 10^{13} \mathrm{~cm}^{-2}$.

- $\mathrm{CO}_{2}$ has an abundance in the range $10^{-6}-10^{-8}$ in the warm atmosphere; its warm column density is $\sim 5 \times 10^{15} \mathrm{~cm}^{-2}$.

- $\mathrm{HCO}$ is more abundant than $\mathrm{CO}_{2}$, with a warm column density of $\sim 1.4 \times 10^{16} \mathrm{~cm}^{-2}$. The warm column density of $\mathrm{HCO}^{+}$is $\sim 1.4 \times 10^{13} \mathrm{~cm}^{-2}$

- $\mathrm{NH}_{3}$ reaches its maximum abundance of $\sim 10^{-6}$ just below the warm region; its warm column is $\sim 3 \times$ $10^{15} \mathrm{~cm}^{-2}$

While $\mathrm{H}_{2} \mathrm{O}$ and some other molecules such as HCN and 
$\mathrm{C}_{2} \mathrm{H}_{2}$ form high up in the atmosphere and then decline in abundance, some species increase further down (e.g., $\left.\mathrm{CH}_{4}\right)$, or at least do not decrease $\left(\mathrm{NH}_{3} ;\right.$ Fig. 2). $\mathrm{CH}_{4}$ has a maximum abundance in the middle of the warm region where $T \sim 800 \mathrm{~K}$. Just below the warm region, it begins to recover from its abundance low $\left(<10^{-10}\right)$ and becomes abundant at large depths: $x\left(\mathrm{CH}_{4}\right) \sim 10^{-7}$ for $N_{\mathrm{H}} \gtrsim 10^{23} \mathrm{~cm}^{-2}$. We find that the chemical as well as the temperature and ionization structure of our disk model is highly stratified. Such a stratified structure is unlikely to be altered by turbulent mixing unless the transport timescale is unusually short (e.g., Heinzeller et al. 2011).

\subsection{Variations on the Reference Case}

In the previous section we presented the abundances of major species obtained for the reference case defined in Table 2. Even for fixed stellar and disk properties, the model is characterized by several parameters: the mechanical (accretion) heating, the dust surface area, the $\mathrm{C} / \mathrm{O}$ ratio and the radial distance. Here we investigate how the variation of these parameters affects the predictions of the model.

One important uncertainty is the efficiency of $\mathrm{H}_{2}$ formation on grains, as discussed at the beginning of Section 2. Without it, i.e., with only gas phase formation of $\mathrm{H}_{2}$, the abundances of molecules other than $\mathrm{CO}\left(\mathrm{H}_{2} \mathrm{O}\right.$, $\mathrm{CO}_{2}, \mathrm{HCN}, \mathrm{C}_{2} \mathrm{H}_{2}$ ) are much reduced in the warm atmosphere, with the result that their warm columns are very small (see also Agúndez et al. 2008; GNI04). As noted in Section 2, theoretical calculations do support $\mathrm{H}_{2}$ formation on warm grains, although there is still considerable uncertainty about the efficiency of this process (e.g., Cuppen et al. 2010). The formation rate also depends on the surface area of the grains, which we parameterize in terms of $a_{g}$ (defined in Sec 2). The grain surface area in the reference model is smaller than the ISM value by a factor of $\sim 30$.

Figure 4 compares the thermal-chemical transition of the reference case (black curves with $a_{g}=0.707 \mu \mathrm{m}$, $\left.\alpha_{h}=1.0\right)$ and two other cases in which $a_{g}$ is increased by a factor of 10 (red curves) and $\alpha_{h}$ is reduced by 100 (blue curves). The red curves correspond to a reduction in the grain surface area per hydrogen nucleus by a factor of $\sim 300$ relative to interstellar grains, and the blue curves have essentially no mechanical heating. In the former case, the transition is shifted significantly deeper down into the disk, from $1 \times 10^{21} \mathrm{~cm}^{-2}$ to $5 \times 10^{21} \mathrm{~cm}^{-2}$, with a corresponding increase in volumetric density. Because the dust cooling rate is also reduced by a factor $\sim 300$, the depth of the warm layer is extended, and the warm molecular columns, given in Table 3, are all increased, with the exception of $\mathrm{OH}$. When mechanical heating is essentially eliminated, the transition is smoother and shifted upwards in the atmosphere to the region $\sim 2 \times 10^{20} \mathrm{~cm}^{-2}-5 \times 10^{20} \mathrm{~cm}^{-2}$. The associated warm region is also shifted upwards, and the warm molecular columns are decreased by a factor of five to ten, with the exception of $\mathrm{OH}$, which is increased by a factor of about three.

From this discussion of we can conclude that warm molecular columns increase with increasing dust size (i.e., reduced dust surface area; parameter $a_{g}$ ) and increasing mechanical heating (parameter $\alpha_{h}$ ). Both effects are ac- companied by a downward shift in the location of the atomic to molecular transition. The warm region starts at the transition, and larger warm columns (which favor detection) are generated as the transition moves downward into regions of higher density. Table 3 gives the warm columns for some of the more abundant species that have either have already been observed in $\mathrm{T}$ Tauri disks or might be candidates for future detection.

$\mathrm{X}$-rays are the only source of external radiation in these model calculations. While varying $T_{\mathrm{X}}$ from $1 \mathrm{keV}$ to $5 \mathrm{keV}$ did not much alter our results, variations in $L_{\mathrm{X}}$ have a much larger effect. Table 4 shows how the warm column of selected molecules and ions depends on $L_{\mathrm{X}}$ at $1 \mathrm{AU}$ for a large range of values of $L_{\mathrm{X}}$ when the other model parameters are kept the same as in the reference case. From Chandra observations of young clusters, Preibisch \& Feigelson (2005) found that the Xray luminosity function depends mildly on cluster age and, within clusters, on the stellar mass range. For the Orion Nebular Cluster, the median K star X-ray luminosity is $L_{\mathrm{X}}=3 \times 10^{30} \mathrm{erg} \mathrm{s}^{-1}$, with $90 \%$ of the stars having $L_{\mathrm{X}}>4 \times 10^{29} \mathrm{erg} \mathrm{s}^{-1}$ and $10 \%$ having $L_{\mathrm{X}}=1.3 \times 10^{31} \mathrm{erg} \mathrm{s}^{-1}$. In Table 4 we can see that the warm columns of ions are especially sensitive to the X-ray luminosity and, as might be expected, increase dramatically as $L_{\mathrm{X}}$ increases. Even for X-ray luminosities as low as $L_{\mathrm{X}}=10^{26} \mathrm{erg} \mathrm{s}^{-1}$ (i.e., several orders of magnitude lower than the X-ray luminosities of typical T Tauri stars), the X-rays are a significant source of ionization, and could influence the chemistry of $\mathrm{T}$ Tauri disks by producing a variety of molecular ions by proton transfer and by destroying neutral molecules by reactions with $\mathrm{H}^{+}$and $\mathrm{He}^{+}$. At these low X-ray ionization rates, the stellar UV radiation field (not treated here) would also be particularly important.

But simple neutral molecules change little as $L_{\mathrm{X}}$ goes from $10^{26}$ to $10^{31} \mathrm{erg} \mathrm{s}^{-1}$. The reason is that mechanical heating associated with the MRI theory of viscosity dominates the heating of the disk atmosphere, and molecular synthesis in the warm regions is built on temperaturesensitive neutral reactions, as discussed in the previous section. In the presence of strong accretion heating, the X-rays also have little effect on the location of the main thermal-chemical transition defined by $\mathrm{CO}$ and $\mathrm{H}_{2}$. For example at $R=1 \mathrm{AU}$, the transition shifts from $6.5 \times 10^{20} \mathrm{~cm}^{-2}$ for $L_{\mathrm{X}}=10^{28} \mathrm{erg} \mathrm{s}^{-1}$ to $1.0 \times 10^{21} \mathrm{~cm}^{-2}$ for $L_{\mathrm{X}}=10^{31} \mathrm{erg} \mathrm{s}^{-1}$, while for $L_{\mathrm{X}}<10^{28} \mathrm{erg} \mathrm{s}^{-1}$ there is no change at all. Nonetheless, molecular ions still play a role, sometimes in forming neutrals by dissociative recombination and sometimes in destroying them by proton transfer or charge transfer reactions.

By way of contrast, radicals are sensitive to X-rays, as exemplified by $\mathrm{OH}$ and $\mathrm{NO}$, both of which increase by about 3,000 when $L_{\mathrm{X}}$ is increased from $10^{26} \mathrm{erg} \mathrm{s}^{-1}$ to $10^{31} \mathrm{erg} \mathrm{s}^{-1}$. In our model, the steady state abundance of $\mathrm{OH}$ in warm regions is mainly determined by its production by recombination of $\mathrm{H}_{3} \mathrm{O}^{+}$and destruction by neutral reactions. $\mathrm{NO}$ follows $\mathrm{OH}$, since it is produced from $\mathrm{OH}$ via Eq. 6 and destroyed by atomic $\mathrm{N}$ via Eq. 7 . A similar explanation applies to $\mathrm{SO}$ and $\mathrm{SO}_{2}$, which are moderately sensitive to X-rays. It is of considerable interest that the warm columns of the hydrocarbons $\mathrm{CH}_{4}$ and $\mathrm{C}_{2} \mathrm{H}_{2}$ decrease with increasing $L_{\mathrm{X}}$. This trend is stronger for $\mathrm{CH}_{4}$, very likely due to the fact that it is 
produced by dissociative recombination of the molecular ion $\mathrm{CH}_{5}^{+}$which is produced by the radiative association of $\mathrm{CH}_{3}^{+}$an $\mathrm{H}_{2}$.

Variations in the $\mathrm{O} / \mathrm{C}$ ratio can have a dramatic effect on the abundances in the warm disk atmosphere. Table 5 gives examples for the reference case at $1 \mathrm{AU}$. The oxygen abundance has been varied at fixed carbon abundance so that the $\mathrm{O} / \mathrm{C}$ ratio varies from 0.25 to 5.0 ; the reference case value is 2.5 . Below $\mathrm{O} / \mathrm{C}=1$, the reduction in the available oxygen leads to a significant decrease in the warm column of $\mathrm{CO}$ and also of $\mathrm{CO}_{2}$. Pure oxygen species such as $\mathrm{O}, \mathrm{OH}, \mathrm{H}_{2} \mathrm{O}, \mathrm{O}_{2}, \mathrm{H}_{3} \mathrm{O}^{+}$show very large decrements, especially below $\mathrm{O} / \mathrm{C}=1$, as do closely related oxides such as $\mathrm{SO}_{2}$ and $\mathrm{NO}$. At the same time, pure carbon species like $\mathrm{C}_{2} \mathrm{H}_{2}$ and $\mathrm{CH}_{4}$ increase drastically. Nitrogen species also change, but by large amounts only when they contain carbon (e.g., HCN) or oxygen (e.g., NO). One noteworthy result of this analysis is that the warm column of $\mathrm{Ne}^{+}$increases rapidly with decreasing $\mathrm{O} / \mathrm{C}$ ratio because it is efficiently destroyed by $\mathrm{H}_{2} \mathrm{O}$.

Ratios of warm columns derived from molecular line flux measurements are often used in making preliminary interpretations of the observations. Our results show that variations in the $\mathrm{O} / \mathrm{C}$ ratio can cause significant changes in the warm column ratios. Because $\mathrm{H}_{2} \mathrm{O}$ decreases more rapidly than $\mathrm{CO}$ with decreasing $\mathrm{O} / \mathrm{C}$, the $\mathrm{H}_{2} \mathrm{O} / \mathrm{CO}$ ratio becomes very small for small $\mathrm{O} / \mathrm{C} \leq 1$. By way of contrast, the $\mathrm{HCN} / \mathrm{H}_{2} \mathrm{O}$ ratio is a sensitive decreasing function of the $\mathrm{O} / \mathrm{C}$ ratio and becomes large as $\mathrm{O} / \mathrm{C}$ approaches unity. The same is true for the $\mathrm{C}_{2} \mathrm{H}_{2} / \mathrm{H}_{2} \mathrm{O}$ ratio, but even more so because the warm column of $\mathrm{C}_{2} \mathrm{H}_{2}$ is a much more sensitive function of $\mathrm{O} / \mathrm{C}$ than HCN. Thus these ratios can be considered as signatures of enhanced carbon chemistry.

Looking at how the overall abundance patterns vary with disk radius, we find that the abundance patterns at 1 AU are similar at smaller and larger radii, as demonstrated in Figure 2 for oxygen molecules (top panel), nitrogen molecules (middle panel) and molecular ions (bottom panel). Some species show large variations in their vertical column density distributions as a function of radius, e.g., $\mathrm{CO}_{2}$. Our results for $40 \mathrm{AU}$ are not plotted because they are qualitatively similar to those for $20 \mathrm{AU}$. Figure 5 and Table 6 show the radial variation of warm columns (i.e., the regions of the disk where line emission can be efficiently generated) for the reference case. The radial range in Figure 5 and Table 6 extends only to 10 AU because the disk atmosphere at 20-40 AU does not have significant column density in the $200-2000 \dot{\mathrm{K}}$ range (Fig. 1). Note that the gas column densities shown in Figures 2 and 5 and Table 6 do not include the effect of freeze-out, which would have a greater impact in the disk midplane.

We see that the warm columns are large at very small radii, decrease rapidly inside $1 \mathrm{AU}$, and then decrease more slowly beyond. This behavior is typified by $\mathrm{H}_{2}$ itself and is shared by many of the more abundant molecules, e.g., $\mathrm{CO}, \mathrm{H}_{2} \mathrm{O}, \mathrm{O}_{2}, \mathrm{NH}_{3}$, and $\mathrm{HCN}$. The water column increases significantly at radii $<0.5$ AU because the neutral reactions that produce water are effective throughout the warm atmosphere (because of the high gas temperature and density in this region) and lead to a high abundance of water throughout (Fig. 2). In contrast, at $1 \mathrm{AU}$, the water abundance drops at intermediate heights (below $N_{\mathrm{H}}=5 \times 10^{21} \mathrm{~cm}^{-2}$ ) before recovering deeper down, leading to a lower warm column than at $0.25 \mathrm{AU}$ (Fig. 5).

It is interesting that, in the top panel of Figure 5, the large warm column of atomic $\mathrm{H}$ changes by no more than a factor of two from $0.25 \mathrm{AU}$ to $10 \mathrm{AU}$. The reason for this is that the basic $\mathrm{OH}$ chemistry operative in the warm region (Eqs. 2 and 4) releases atomic $\mathrm{H}$ as atomic oxygen is processed into water. The abundance of atomic $\mathrm{H}$ is important for the synthesis of hydrocarbon molecules because many of the reactions that hydrogenate carbon are reversed by atomic $H$ reactions.

Many of the heavier molecules show similar patterns because they share a common (warm neutral radical) chemistry and because the physical properties of the warm region change slowly with disk radius. For example, the ionization parameter has the value $\zeta / n_{\mathrm{H}} \sim$ $3 \times 10^{-20} \mathrm{~cm}^{3} \mathrm{~s}^{-1}$ to within a factor of two over radii from 0.25 to $20 \mathrm{AU}$ (a factor of almost 100) because the inverse square dilution of the X-ray ionization rate (top panel of Fig. 1) is largely compensated for by the decrease of the density with radius. Consequently the electron fraction at the top of the warm region is almost independent of radius, with a value close to $x_{\mathrm{e}}=2 \times 10^{-6}$. This approximate invariance applies to the ionization of the warm region, but not to the molecular abundances which are affected by density and temperature (and processes such as freeze out) as well as ionization. The temperature does remain close to $1000 \mathrm{~K}$ at the top of the warm column for small radii (middle panel of Fig. 1), but it then decreases significantly beyond $4 \mathrm{AU}$. Of course the density at a given vertical column density decreases by more than 1,000 as the radius increases from 0.25 to $20 \mathrm{AU}$. These changes also cause the thermal chemical transition to move slightly upward in the atmosphere with increasing radius, and this also tends to lead to a reduction in warm column densities. The decreasing density of the warm atmosphere as a function of radius may play a role in limiting the radial extent of emission from molecular transitions with high critical densities.

\section{DISCUSSION}

\subsection{Inner Planet Formation Region}

Our model results (Section 3) have potential implications for the understanding of the inner and outer planet formation regions of the disk, i.e., the regions roughly within and beyond the "snow line" where water vapor would condense on grains. The inner planet formation region, discussed in this section, is probed by the molecular observations made with Spitzer and ground-based near-infrared spectroscopy. The outer planet formation region is discussed in the next section.

\subsubsection{Warm Molecular Columns}

As we found in GMN09, the warm atmosphere in our models achieves temperatures comparable to that of the Spitzer molecular emission from T Tauri disks $(\sim 300$ 1000 K; Carr \& Najita 2008, 2011; Salyk et al. 2008, 2011). These temperatures are maintained over significant column densities at disk radii out to $\sim 4 \mathrm{AU}$ (Figure 1, middle panel). Thus the temperature structure of our disk atmosphere is consistent with the interpretation that the Spitzer molecular emission arises from the inner 
few AU of the disk.

Some molecular species appear quite sensitive to the parameter variations we considered, with their warm molecular columns varying significantly over the parameter range we explored. As one example, the variation in grain size and mechanical heating alone can produce warm column variations of $\sim 30$ in $\mathrm{H}_{2} \mathrm{O}$ and $\mathrm{HCN}$ (Table 3 ). This sensitivity may help to account for the factor of $\sim 30$ range in the $\mathrm{H}_{2} \mathrm{O}$ and $\mathrm{HCN}$ emission strengths among T Tauri disks (Pontoppidan et al. 2010; Salyk et al. 2011; Carr \& Najita 2011). The role of grain settling in producing enhanced warm columns may also help to account for the finding by Salyk et al. (2011) of the enhanced detectability of molecular emission from more settled disks.

The warm molecular columns we find for some species are also quantitatively similar to those inferred from the Spitzer data. The line-of-sight column density of the water emission detected with Spitzer $\left(\sim 10^{18} \mathrm{~cm}^{-2}\right)$, which has an associated projected emitting area of $\pi r^{2}$ where $r \sim 1$ AU (Carr \& Najita 2011; see also Salyk et al. 2011 ), is similar to our (vertical) warm column of water at $1 \mathrm{AU}\left(10^{17}-5 \times 10^{18} \mathrm{~cm}^{-2}\right.$; Tables 3,4 , and 5$)$. The much smaller warm columns achieved at larger disk radii $\left(<10^{17} \mathrm{~cm}^{-2}\right.$ at $10 \mathrm{AU}$ in the reference case) help to account for the inference of AU-sized water emission regions from the Spitzer spectra, although perhaps not completely (see Section 4.2).

Larger warm columns are achieved at small disk radii $\left(\sim 10^{20} \mathrm{~cm}^{-2}\right.$ at $0.25 \mathrm{AU}$ in the reference case; Table 6 and Figure 5). These are reminiscent of the water columns reported at such radii based on $K$ - and $L$-band spectroscopy of disks $\left(\sim 10^{20}-10^{21} \mathrm{~cm}^{-2}\right.$; Carr et al. 2004; Najita et al. 2009; Doppmann et al. 2011). However, the temperature of the observed emission is warmer ( 1500 K) than most of the warm column $(2000-200 \mathrm{~K})$ in the model. Thus, while the empirical situation is that strong near-infrared water emission is rare (e.g., Najita et al. 2007), this does not necessarily mean that water is underabundant at small disk radii in most disks. Our models suggest that large columns of water are commonly present at small disk radii, but the water is typically not warm enough to produce strong water emission in the near-infrared.

We can also compare our results to those derived for the $4.7 \mu \mathrm{m}$ CO fundamental emission from T Tauri stars, which has a characteristic temperature of $\sim 500-1000 \mathrm{~K}$, line-of-sight column density $\sim 10^{18} \mathrm{~cm}^{-2}$, and projected emitting area of radius $\sim 0.15$ AU (Salyk et al. 2011; Najita et al. 2003). Our reference model at 0.25 AU produces a vertical CO column of $5 \times 10^{17} \mathrm{~cm}^{-2}$ over the vertical temperature range $2000-500 \mathrm{~K}$, in reasonable agreement with the observed values. The decrease with radius of both the density of the atmosphere and CO column over this temperature range may contribute to limiting the radial extent of the $\mathrm{CO}$ fundamental emission from the disk.

The Spitzer HCN emission detected from T Tauri stars has been characterized as having a typical temperature of $\sim 700 \mathrm{~K}$ (Carr \& Najita 2011; Salyk et al. 2011). If the emitting area is assumed to be the same as that of the water emission (i.e., $\sim 1 \mathrm{AU}$ ), the line-of-sight column density is typically $\sim 10^{15} \mathrm{~cm}^{-2}$ (Salyk et al. 2011).
Fits made without that assumption yield smaller emitting areas $(0.3-0.6 \mathrm{AU})$ and larger HCN columns (a few $\times 10^{16} \mathrm{~cm}^{-2}$ ), although the lower columns of Salyk et al. are not ruled out (Carr \& Najita 2011). In our models, $\mathrm{HCN}$ is abundant throughout the inner disk atmosphere, with a warm column of $10^{15}-10^{16} \mathrm{~cm}^{-2}$ at $1 \mathrm{AU}$ depending on the model parameters (Tables 3, 4, and 5), a range that encompasses the values inferred by Salyk et al. (2011). The larger line-of-sight columns preferred by Carr \& Najita (2011) may not be too different from the warm (vertical) columns of our models if the HCN emission arises at disk radii closer to $0.5 \mathrm{AU}$, where the warm column is larger, and when disk inclination effects are taken into account.

The similar temperatures found for the Spitzer $\mathrm{HCN}$ and water emission (Carr \& Najita 2008, 2011; Salyk et al. 2008, 2011) suggest that the HCN emission arises from the same region of the disk atmosphere as the water emission. The smaller emitting area inferred for the HCN emission, compared to the water emission, in the best-fit models of Carr \& Najita $(2008,2011)$ might seem odd in that context. The difference might indicate that the $\mathrm{HCN}$ emission is restricted to a smaller (possibly annular) region than the water emission, perhaps because of the molecular abundance distribution or excitation considerations. In our models, the smooth variation of the warm HCN column with radius and the slow decline beyond $1 \mathrm{AU}$ (Figure 5) does not suggest an annular distribution for the HCN. However, the radial range of the HCN emission may also be restricted by the high densities needed to excite the observed transitions and the decline in density with disk radius in the warm atmosphere (Figure 2).

The $\mathrm{C}_{2} \mathrm{H}_{2}$ emission detected with Spitzer has been characterized as having a $\mathrm{C}_{2} \mathrm{H}_{2} / \mathrm{HCN}$ column density ratio of $\sim 0.1$ and a $\mathrm{C}_{2} \mathrm{H}_{2}$ column of $10^{15}-10^{16} \mathrm{~cm}^{-2}$, assuming that the $\mathrm{C}_{2} \mathrm{H}_{2}$ emission has the same temperature and emitting area as the $\mathrm{HCN}$ emission (Carr \& Najita 2008, 2011). Salyk et al. (2011) instead assumed that the $\mathrm{C}_{2} \mathrm{H}_{2}$ and $\mathrm{HCN}$ emission have the same emitting area as the water emission but allowed the $\mathrm{C}_{2} \mathrm{H}_{2}$ and $\mathrm{HCN}$ temperatures to differ; they inferred typical $\mathrm{C}_{2} \mathrm{H}_{2} / \mathrm{HCN}$ column ratios $\sim 1$ and columns of $\sim 10^{15} \mathrm{~cm}^{-2}$ for $\mathrm{C}_{2} \mathrm{H}_{2}$. In our reference model, the $\mathrm{C}_{2} \mathrm{H}_{2} / \mathrm{HCN}$ warm column ratio is $\sim 0.01$ and the $\mathrm{C}_{2} \mathrm{H}_{2}$ column density is $\sim 10^{13} \mathrm{~cm}^{-2}$ at $1 \mathrm{AU}$, both of which are smaller than indicated by the observations. However, for the case $a_{g}=7.07$, the $\mathrm{C}_{2} \mathrm{H}_{2} / \mathrm{HCN}$ warm column ratio is $\sim 0.1$ and the $\mathrm{C}_{2} \mathrm{H}_{2}$ column density is $\sim 10^{15} \mathrm{~cm}^{-2}$ at 1 AU (Table 3 ), more similar to the reported values.

The Spitzer $\mathrm{CO}_{2}$ emission has been fit with characteristic temperatures higher (Salyk et al. 2011) or lower than (Carr \& Najita 2011) that of water. Carr \& Najita (2011) find that the $\mathrm{CO}_{2}$ column density is not well constrained by the observations. Salyk et al. (2011) report $\mathrm{CO}_{2}$ column densities $\sim 10^{15}-10^{16} \mathrm{~cm}^{-2}$, assuming the $\mathrm{CO}_{2}$ emission has the same emitting area as the $\mathrm{H}_{2} \mathrm{O}$ emission. In our models, the $\mathrm{CO}_{2}$ warm column is $\sim 10^{15}-10^{16} \mathrm{~cm}^{-2}$ at 1 AU (Tables 3, 4, and 5), similar to the reported values.

As in GMN09, our models are unable to account for the $\mathrm{OH}$ emission from inner disks. The observed $\mathrm{OH}$ columns are $3 \times 10^{14}-3 \times 10^{16} \mathrm{~cm}^{-2}$ (Salyk et al. 2011; Carr \& Na- 
jita 2008), higher than in our models $\left(\sim 10^{14}-10^{15} \mathrm{~cm}^{-2}\right.$; Tables 3, 4, and 5). The larger $\mathrm{OH}$ columns are very likely the result of UV irradiation of the disk, which is not yet implemented here. UV irradiation can dissociate $\mathrm{H}_{2} \mathrm{O}$ to produce $\mathrm{OH}$, potentially explaining the large $\mathrm{OH}$ columns that are observed (Bethell \& Bergin 2009; GMN09). Hot OH emission, which may result from the photodissociation of $\mathrm{H}_{2} \mathrm{O}$ (e.g., Najita et al. 2010), has been reported from T Tauri disks (Carr \& Najita 2011), consistent with this interpretation. Given the central role of the $\mathrm{OH}$ radical in the warm chemistry of disk atmospheres (Figure 3), an enhanced $\mathrm{OH}$ abundance (e.g., from photodissociation) may lead to enhanced abundances of species that are synthesized from $\mathrm{OH}$.

Our models address the question raised by Salyk et al. (2011) regarding the dominant carrier of nitrogen in the disk. Noting that the HCN abundance they infer does not account for all of the available nitrogen, Salyk et al. (2011) suggested $\mathrm{NH}_{3}$ or $\mathrm{N}_{2}$ as the possible carriers of the remaining nitrogen. They also pointed out how $\mathrm{NH}_{3}$ is unlikely to be the missing reservoir: given the upper limit on $\mathrm{NH}_{3}$ emission in the Spitzer spectra in the $10 \mu \mathrm{m}$ region, they inferred that the column of warm $\mathrm{NH}_{3}$ is $<10^{16} \mathrm{~cm}^{-2}$, and $\mathrm{N}_{2}$ was suggested to be the dominant reservoir of nitrogen. Consistent with this interpretation, our model predicts warm $\mathrm{NH}_{3}$ columns of $10^{15}-10^{16} \mathrm{~cm}^{-2}$ at $1 \mathrm{AU}$ (Tables 3, 4, and 5) and that most of the nitrogen is in $\mathrm{N}_{2}$.

\subsubsection{Molecular Emission Trends}

Our models may also provide some context for the various molecular emission trends that have been reported in the literature. In their study of a small sample of T Tauri stars, Carr \& Najita (2011) found tentative evidence that the flux ratio of $\mathrm{HCN} / \mathrm{H}_{2} \mathrm{O}$ emission increases with disk mass, increasing by a factor of $\sim 4$ over disk masses of $0.002-0.02 M_{\odot}$. They interpreted this result as a possible consequence of planetesimal or protoplanet formation. This interpretation arises from considering the transport of water in the solar nebula (Ciesla \& Cuzzi 2006). Because icy bodies ranging from micron, to meter, to kilometer and larger size migrate at different rates, they can transport water ice to the inner disk at greater or lesser rates compared to the gas, reducing or enhancing the $\mathrm{O} / \mathrm{C}$ ratio of the inner disk and possibly affecting the chemistry of the inner disk.

That is, when grains in the outer planet formation region are small enough to couple well to the gas, water that is frozen on these grains accretes along with the gas into the inner planet formation region. The water is returned to the gas phase when the ices evaporate, and the $\mathrm{O} / \mathrm{C}$ ratio of the inner disk gas is representative of the disk as a whole. However, when the icy grains grow into meter-sized bodies, they migrate inward extremely rapidly relative to the gas. When they evaporate, they hydrate the inner disk, and in depositing water and oxygen potentially enhance the $\mathrm{O} / \mathrm{C}$ ratio there. In contrast, if icy material in the outer planet formation region grows into planetesimal or larger bodies, such large bodies cease to migrate (and may go on to form planets). They thereby sequester water (and oxygen) in the outer planet formation region, leaving the inwardly migrating material dehydrated and oxygen-poor (e.g., Ciesla
\& Cuzzi 2006). This situation might both reduce the water abundance in the inner disk and, by reducing the $\mathrm{O} / \mathrm{C}$ ratio, favor the formation of hydrocarbons in the inner disk.

We might therefore expect that early in the disk evolution process (before significant grain growth has occured) the gaseous $\mathrm{O} / \mathrm{C}$ ratio of the inner planet formation region will reflect the bulk $\mathrm{O} / \mathrm{C}$ ratio of the disk. At intermediate times, the inner disk may have a higher $\mathrm{O} / \mathrm{C}$ than the disk as a whole. At late times, the inner disk may have a lower $\mathrm{O} / \mathrm{C}$ ratio than the disk as a whole. The timescale on which a disk transitions from "early" to "intermediate" to "late" depends on the disk mass: more massive disks would transition more rapidly through each phase.

Thus, a coeval cluster of $\mathrm{T}$ Tauri stars with a range of disk masses may have inner disks with a range of gasphase $\mathrm{O} / \mathrm{C}$ ratios, and we might expect the inner disks of $\mathrm{T}$ Tauri stars to show a range of $\mathrm{HCN} / \mathrm{H}_{2} \mathrm{O}$ flux ratios, as is observed (Carr \& Najita 2011; Salyk et al. 2011). For T Tauri stars transitioning from the "intermediate" to "late" phase, $\mathrm{HCN} / \mathrm{H}_{2} \mathrm{O}$ might increase with disk mass, possibly accounting for the tentative trend reported by Carr \& Najita (2011).

Our results support this scenario. As expected, a lower $\mathrm{O} / \mathrm{C}$ ratio enhances the $\mathrm{HCN}$ abundance and decreases the $\mathrm{H}_{2} \mathrm{O}$ abundance in the inner disk. A very modest decrease in the $\mathrm{O} / \mathrm{C}$ ratio, from 2.5 (our reference case) to $\sim 1.8$ would raise the $\mathrm{HCN} / \mathrm{H}_{2} \mathrm{O}$ ratio of warm columns by a factor of $\sim 4$. It would be interesting to measure other molecular ratios that might corroborate this picture. We had earlier suggested the possibility of using $\mathrm{C}_{2} \mathrm{H}_{2} / \mathrm{H}_{2} \mathrm{O}$ as a diagnostic of the $\mathrm{O} / \mathrm{C}$ ratio (Section 3 ). Our results also suggest diagnostics of the "intermediate" phase of evolution. For the case of $\mathrm{O} / \mathrm{C}$ higher than the reference model (i.e., an $\mathrm{O} / \mathrm{C}$ ratio of 5), oxygen-bearing molecules such as $\mathrm{H}_{2} \mathrm{O}$ and $\mathrm{O}_{2}$ are enhanced and carbonbearing molecules such as $\mathrm{C}_{2} \mathrm{H}_{2}$ and $\mathrm{HCN}$ are reduced in abundance relative to the reference case (Table 5).

An alternative explanation for the tentative trend of $\mathrm{HCN} / \mathrm{H}_{2} \mathrm{O}$ emission with disk mass reported by Carr \& Najita (2011) may be an excitation effect, where HCN is better excited at the higher densities that would be present in a more massive disk. This scenario would require that the disk mass estimated from observations that probe large disk radii ( $>20 \mathrm{AU}$ from submillimeter/millimeter observations) apply to the inner few $\mathrm{AU}$ of disks. These two possibilities $(\mathrm{O} / \mathrm{C}$ ratio or excitation effect) could be tested by looking for trends with disk mass of emission from other molecules that are less sensitive to density. Detailed excitation modeling would also be useful in sorting out these possibilities.

More perplexing is the report by Teske et al. (2010) of a possible trend of HCN flux with $L_{\mathrm{X}}$, with $\mathrm{HCN}$ flux increasing on average by a factor of $\sim 4$ in the range $L_{\mathrm{X}}=2 \times 10^{29}-2 \times 10^{30} \mathrm{erg} \mathrm{s}^{-1}$. In our models, a higher $L_{\mathrm{X}}$ enhances the abundance of $\mathrm{He}^{+}$, which can break up $\mathrm{CO}$ and potentially enhance the HCN abundance. However, our warm HCN columns vary negligibly with $L_{\mathrm{X}}$ in the $\mathrm{T}$ Tauri luminosity range $\left(L_{\mathrm{X}}=10^{28}-10^{31} \mathrm{erg} \mathrm{s}^{-1}\right)$. It would be interesting to see if studies of larger samples of objects confirm a trend of HCN flux with $L_{\mathrm{X}}$.

There is a bigger difference between the warm HCN column for the reference case and for very low values of 
$L_{\mathrm{X}}$. For $L_{\mathrm{X}}=10^{26} \mathrm{erg} \mathrm{s}^{-1}$, the warm HCN column is an order of magnitude larger than in the reference case (Table 4). As T Tauri stars are known to be strong emitters of X-rays at a much higher level than $10^{26} \mathrm{erg} \mathrm{s}^{-1}$, the ions produced by $\mathrm{T}$ Tauri X-ray emission appear to have the effect of limiting the $\mathrm{HCN}$ abundance and emission from disks.

There is tentative evidence that $\mathrm{HCN}$ flux increases with stellar accretion rate, with $\mathrm{HCN}$ flux increasing from $<1 \mathrm{mJy}-\mu \mathrm{m}$ to $\sim 4 \mathrm{mJy}-\mu \mathrm{m}$ over the range $10^{-9}-$ $10^{-7} M_{\odot} \mathrm{yr}^{-1}$ (Teske et al. 2010). One possible explanation for this is that the increased HCN flux arises from increased heating. GMN09 had earlier discussed how enhanced accretion-related mechanical heating in the disk atmosphere could enhance the water emission from the disk, primarily by increasing the column density of the warm atmosphere. We would expect a similar enhancement in HCN emission, since a warmer disk atmosphere would enhance the emission from other molecules as well as water, all other things being equal.

Our results show that indeed increased accretionrelated heating enhances the column of warm $\mathrm{HCN}$ in the disk atmosphere. The warm HCN column increases by a factor of $\sim 3\left(\right.$ from $2.4 \times 10^{14} \mathrm{~cm}^{-2}$ to $7.7 \times 10^{14} \mathrm{~cm}^{-2}$ ) for values of $\alpha_{h}=0.01$ to 1 , with most of the increase in column occuring in the range $\alpha_{h}=0.1-1$. In the higher $\alpha_{h}$ case, the warm HCN also occurs deeper in the atmosphere and at higher density where it may be better excited. If higher stellar accretion rates are accompanied by higher rates of mechanical heating in the disk atmosphere, both the increase in the warm column of $\mathrm{HCN}$ and the higher density region in which it is located may account for the increased HCN flux that is observed at higher stellar accretion rates.

An alternative explanation is that the higher UV fluxes associated with accretion lead to greater grain photoelectric heating and enhanced warm molecular columns. Detailed modeling is needed to explore the impact of increased UV irradiation and whether the higher heating and photodissociation rates lead to higher or lower warm molecular columns. The result is likely to depend on the degree of grain settling in the atmosphere (e.g., Nomura et al. 2007).

An alternative chemical explanation for the trend is that the higher UV fluxes dissociate $\mathrm{N}_{2}$ in favor of atomic $\mathrm{N}$, which then enhances the formation and abundance of HCN. Such a scenario was discussed by Pascucci et al. (2009) who argued that the higher flux ratio of $\mathrm{HCN} / \mathrm{C}_{2} \mathrm{H}_{2}$ in the spectra of $\mathrm{T}$ Tauri stars compared to those of brown dwarfs may result from the higher accretion rates and consequently higher UV fluxes of $\mathrm{T}$ Tauri stars. In our models, a significant fraction (approximately half) of the nitrogen is already in atomic form in the warm atmosphere even in the absence of UV irradiation, with the remainder primarily in $\mathrm{N}_{2}$. Including the UV dissociation of $\mathrm{N}_{2}$ may then have a limited impact on the $\mathrm{HCN}$ abundance, although detailed calculations are needed to be certain.

\subsubsection{Water Molecule Shielding and Heating}

The column density over which UV irradiation affects the disk atmosphere, either chemically (through photodissociation) or thermally (by depositing energy into the atmosphere), is governed by the abundance of UV- absorbing dust and molecules in the disk atmosphere. Molecules may provide the dominant source of UV opacity in T Tauri disk atmospheres given the high inferred rate of dust settling from disk atmospheres, as previously noted in the specific context of water by Bethell \& Bergin (2009).

Our results support this interpretation. Since water absorbs UV photons in the $1200-1800 \AA$ range with a cross-section of $10^{-17}-10^{-18} \mathrm{~cm}^{2}$ (Parkinson \& Yoshino 2003), stellar UV photons encounter an optical depth of unity over a line-of-sight disk column density (to the star) of $N_{\mathrm{H}_{2} \mathrm{O}} \sim 10^{17}-10^{18} \mathrm{~cm}^{-2}$, or a vertical column density that is $\sim 10$ times smaller. The much larger total vertical water column densities that can be synthesized in our disk atmosphere models $\left(\gg 10^{18} \mathrm{~cm}^{-2}\right)$, the warm column component of which $\left(\sim 10^{18} \mathrm{~cm}^{-2}\right)$ is observed in emission from T Tauri disks (Carr \& Najita 2008, 2011; Salyk et al. 2008, 2011), appear to be capable of shielding the lower disk atmosphere and the disk midplane from UV irradiation.

As noted above, UV irradiation of the disk may also heat the disk surface through the absorption of UV photons by water and other molecules. A simple estimate of the heating rate per unit volume due to the absorption of UV photons by water is

$$
\Gamma_{\mathrm{UV}}=\zeta_{*} e^{-\tau_{\mathrm{uv}}} E_{\mathrm{UV}} x_{\mathrm{H}_{2} \mathrm{O}} n_{H},
$$

where $\zeta_{*}$ is the unshielded dissociation rate due to stellar UV, $\tau_{\mathrm{uv}}$ is the attenuation of the UV along a line of sight to a given position in the disk, and $E_{\mathrm{UV}}$ is the energy deposited as heat per dissociation. Laboratory experiments show that water is strongly dissociated in

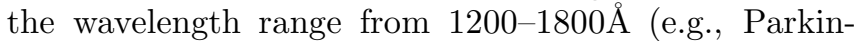
son \& Yoshino 2003). Since the mean photon wavelength for dissociation is $\sim 1500 \AA$ and the threshold is $2340 \AA, E_{\mathrm{UV}} \simeq 3 \mathrm{eV}$. Bergin et al. (2003) showed that the stellar UV flux of the representative $\mathrm{T}$ Tauri star BP Tau at a distance of $100 \mathrm{AU}$ is $\sim 540$ times the standard Habing interstellar radiation field, or $\sim 5.4 \times 10^{6}$ larger at $1 \mathrm{AU}$. Multiplying the unattenuated interstellar photodissociation rate of $5.9 \times 10^{-10} \mathrm{~s}^{-1}$ (Roberge et al. 1991) by $3 \times 10^{6}$, after correcting for the use by Roberge et al. of the Draine interstellar radiation field, yields $\zeta_{*}=1.8 \times 10^{-3} \mathrm{~s}^{-1}$.

Similarly, the $\mathrm{X}$-ray heating rate is

$$
\Gamma_{\mathrm{X}}=\zeta_{\mathrm{X}} e^{-\tau_{\mathrm{x}}} E_{\mathrm{X}} n_{H},
$$

where $\zeta_{\mathrm{X}}$ is the unshielded $\mathrm{X}$-ray ionization rate, $\tau_{\mathrm{x}}$ is the line of sight $\mathrm{X}$-ray attenuation factor, and $E_{\mathrm{X}}$ is the heating per X-ray ionization. In our reference model, $\zeta_{\mathrm{X}}=8 \times 10^{-9} \mathrm{~s}^{-1}$ at the top of the atmosphere at $1 \mathrm{AU}$, and $E_{\mathrm{X}} \sim 20 \mathrm{eV}$ (GNI04). Substituting the given numbers and assuming that $x\left(\mathrm{H}_{2} \mathrm{O}\right)=10^{-4}$, the ratio of the UV water to X-ray heating rates is $\sim 3 e^{-\tau_{\mathrm{uv}}} / e^{-\tau_{\mathrm{x}}}$. This rough estimate confirms that UV water heating is potentially significant at the disk surface and that it is sensitive to the absorption of the UV by dust and by water itself. In the reference model of this paper at $1 \mathrm{AU}$, accretion heating in the neighborhood of the transition region dominates X-ray heating by a factor between 10 and 100. A UV heating rate that is $\sim 3$ times the X-ray heating rate could make a quantitative difference, especially where the transition occurs. 
Thus water in the disk atmosphere may heat itself (cf. Bethell \& Bergin 2009), a situation that would help to explain why the water emission observed with Spitzer has similar properties from source to source (Carr \& Najita 2011). If water is a significant heat source at the disk surface, and if $\mathrm{HCN}$ is abundant at the disk surface (e.g., in the low $L_{\mathrm{X}}$ case or the case of $a_{g}=7.07 \mu \mathrm{m}$ ), UV heating by water may have a (thermal) role in explaining a trend between HCN flux and stellar accretion rate. In addition to its potential role as a heat source, water is also known to be an important coolant based on its observed emission luminosity (Pontoppidan et al. 2010). It would be important to explore the impact of water on both the heating and cooling of the disk atmosphere. Similar considerations apply to FUV absorption by $\mathrm{H}_{2}$, which is more abundant than $\mathrm{H}_{2} \mathrm{O}$ but absorbs over a more limited wavelength region. A detailed investigation is needed to determine how and where these processes affect the disk properties.

\subsection{Outer Planet Formation Region}

The outer planet formation region, with its low dust temperature (e.g., < $150 \mathrm{~K}$ at $10 \mathrm{AU}$; Fig. 1), is more difficult to treat with our current model because we do not include freeze out and desorption processes. Moreover, the lower gas temperatures and densities in the outer planet formation region (Fig. 1) lead to slower chemical timescales. As a result, the steady state abundances that we calculate may be less valid, and transport processes (e.g., Willacy et al. 2006; Willacy \& Woods 2009; Nomura et al. 2009; Heinzeller et al. 2011), which are not treated here, important. Nevertheless, some aspects of our results may bear on existing observations and future attempts to observe this region of the disk.

Our simple models predict a dramatic decrease in the warm column of water beyond a few AU. At $10 \mathrm{AU}$ and beyond, much of the water that is produced would freeze out at the low grain temperatures in the atmosphere. However, the model does predict a detectable water column at smaller radii in the region of the disk atmosphere where the dust temperature is high enough that water would not freeze out. At $4 \mathrm{AU}$, the column of water above the point where the grain temperature reaches $150 \mathrm{~K}$, $125 \mathrm{~K}$, and $100 \mathrm{~K}$ is $2 \times 10^{16} \mathrm{~cm}^{-2}, 2 \times 10^{17} \mathrm{~cm}^{-2}$, and $3 \times 10^{17} \mathrm{~cm}^{-2}$ respectively. Such a warm water column would likely produce detectable emission, in potential conflict with the Spitzer results, which find a small emitting area for the water emission of $\pi r^{2}$ where $r<2 \mathrm{AU}$ (Carr \& Najita 2011; Salyk et al. 2011). This point was made by Meijerink et al. (2009) based in part on the assumptions of the GMN09 model.

Meijerink et al. suggested vertical transport as a way of resolving the potential conflict (see also Pontoppidan et al. 2010). In the scenario they proposed, water from the disk surface is transported, via vertical turbulent diffusion, further down in the disk to a region where the dust temperature is low enough that water can freeze out onto the grains. These icy grains settle to the disk midplane and are incorporated into large enough bodies that they are not relofted to the disk surface. Meijerink et al. argue that this effect (a "cold finger"), can account for the small emitting area inferred from the Spitzer water observations. They further suggested that the radial distribution of water emission at the disk surface might trace the location of the midplane snowline, which is of interest for planet formation.

Since this picture, created to account for the properties of the water emission observed with Spitzer, would also apply to other molecules with similar condensation temperatures, one way to test the picture is to see if such molecules have a similar radial distribution to that of water. One possible test molecule is $\mathrm{HCN}$; its condensation temperature is similar to that of water (e.g., Walsh et al. 2010) and our models predict it to remain abundant in the disk atmosphere out to $10 \mathrm{AU}$ or more in the absence of freeze out. Thus far, the emitting areas inferred for HCN emission from the Spitzer data are not sufficiently constrained to test this picture, as the HCN area can be smaller than, similar to, or greater than the water emitting area, given the uncertainties (Carr \& Najita 2011). Searches for emission from cooler water and HCN, e.g., with the Herschel Space Telescope, may better address this issue.

There are several considerations that would bear on whether our model results really motivate a "cold finger" for water or any other molecule, as differences between the model results and observations might be accounted for, to some extent, by some combination of the following considerations. Firstly, the disk atmosphere beyond a few AU may be cooler than we have inferred. If grains have settled less in the outer disk (e.g., than in the inner disk), we will have underestimated the gas-grain cooling there. Another concern is that we have neglected water cooling. Including such effects may reduce the warm water column and the molecular emission from the disk atmosphere without requiring vertical transport. Secondly, the synthesis of molecules such as water may be less efficient than we have assumed. Some of the neutral reactions we have used have thermal barriers and their rate coefficients are very sensitive to temperature (e.g., eqs. 2 and 4) and may have been overestimated in the analyses of low temperature laboratory data. Another important issue is the (uncertain) efficiency of $\mathrm{H}_{2}$ formation on grains at high temperature, as discussed in Section 3. Thirdly, photodissociation may limit the amount of water that is present in the disk atmosphere. While processes such as photodissociation and a reduced efficiency of $\mathrm{H}_{2}$ formation on grains will affect both the inner and outer disks, they may have a greater relative impact on the outer disk (where the warm water column is expected to be small even without these effects; Fig. 5) and may push the warm water column below an observable level in the outer disk.

We can test the first point above by looking for molecular emission from molecules other than water. Our model predicts a thermal-chemical transition out to beyond $40 \mathrm{AU}$ and that $\mathrm{CO}$ is abundant in the disk atmosphere over that range of radii. The presence or absence of any resulting warm CO emission (e.g., with the Herschel Space Telescope) would probe whether or not a warm disk atmosphere is present at radii beyond a few AU.

How well can the models presented here really identify chemical signatures of planetesimal formation, one of the longer-term motivations for our study? Our variation of the $\mathrm{O} / \mathrm{C}$ ratio in the inner planet formation region of the disk may capture some of the relevant behavior of the scenario discussed in section 4.1.2. However, a 
detailed freeze out and desorption model and the inclusion of transport processes (e.g., as explored by other authors) is likely needed to predict which species remain in the disk atmosphere and at what abundance. There are a number of uncertainties in constructing such a model (see e.g., Walsh et al. 2010 for a discussion of desorption processes). This is an important area for future work.

One tracer of the disk atmosphere that is independent of many of the above concerns is HI $21 \mathrm{~cm}$ radiation, which is of interest because one feature of X-ray irradiated disk atmospheres is a significant $\mathrm{HI}$ component. Our models indicate an HI column of $2 \times 10^{17}-7 \times 10^{17} \mathrm{~cm}^{-2}$ in the 1-20 AU region from each face of the disk. A similar column extending to $100 \mathrm{AU}$ would imply an HI mass of $\sim 10^{28} \mathrm{gm}$, a value that is consistent with current HI upper limits but may be detectable with future observational facilities (Kamp et al. 2007).

\section{SUMMARY AND FUTURE DIRECTIONS}

As discussed in the introduction, one goal of our modeling effort was to explore the potential role of processes such as grain settling, X-ray irradiation, accretionrelated mechanical heating, and the $\mathrm{O} / \mathrm{C}$ ratio in determining the thermal-chemical properties of disk atmospheres. Another was to explore how well a model that excludes UV irradiation can do in accounting for the existing observations of inner disk atmospheres. We also aimed to make detailed comparisons with the observations in order to determine the changes needed to improve our model.

To address these goals, we used an expanded version of our thermal-chemical model of disk atmospheres to explore the sensitivity of the properties of the atmosphere to model parameters. We find that the warm columns (200-2000 K) of many molecular species are sensitive to grain settling and the efficiency of accretion heating, primarily because these parameters affect the depth of the warm molecular layer at the disk surface. We also showed how certain model parameters (grain growth, stellar Xray luminosity, mechanical heating, $\mathrm{O} / \mathrm{C}$ ratio) affect the molecular abundances of disk atmospheres, and which species are sensitive to which parameters.

We find many areas of agreement with the observations. The model parameter variations we considered can account for many of the properties of molecular species that have been detected with Spitzer and ground-based near-infrared spectroscopy (i.e., their typical temperatures, warm columns, and emitting areas). The sensitivity of warm columns to parameters such as grain settling and accretion heating may account, at least in part, for the large range in the molecular emission fluxes that have been observed from $\mathrm{T}$ Tauri disks.

The dependence of the warm columns on model parameters such as grain growth, accretion heating, and $\mathrm{O} / \mathrm{C}$ ratio, may help to explain the trends reported in the literature between the detection rate of molecular emission and mid-infrared color (Salyk et al. 2011) and molecular emission flux with stellar accretion rate (Teske et al. 2011) and disk mass. Regarding the last, we find that the ratio of $\mathrm{HCN} / \mathrm{H}_{2} \mathrm{O}$ increases with a decreasing $\mathrm{O} / \mathrm{C}$ ratio; a decreasing $\mathrm{O} / \mathrm{C}$ ratio in the inner disk is a potential effect of planetesimal and protoplanet formation in the outer disk, which likely occurs at an accelerated rate in higher mass disks (Carr \& Najita 2011).
While the parameter variations we explored can account for the average values and ranges of the observed molecular emission properties of disks, a future challenge will be to explain the properties of individual sources with a single set of model parameters. Our model suffers from some obvious limitations; e.g., our low $\mathrm{OH}$ abundance points out the need for including UV irradiation and photodissociation in our model. The results of our steady-state chemical model are also likely less valid in the outer planet formation region of the disk because of the longer chemical timescales there and because we have not included freezeout, desorption and transport processes. We aim to address these challenges in future work.

We are grateful to John Carr for a careful reading of the manuscript. This work has been supported in part by NASA grant NNG06GF88G (Origins) and by NASA Herschel contracts 132594 (Theoretical Research) and 1367693 (DIGIT) to UC Berkeley.

Ádámkovics, M., Glassgold, A. E., \& Meijerink, R. 2011, ApJ, 736, 143 (AGM11)

Agúndez, M., Cernicharo, J., \& Goicoechea, J. R. 2008, A\&A, 483, 831

Anicich, V. G. 1993, J. Phys. Chem. Ref. Data, 22, 1469 Asplund, M., Grevesse, N., Sauval, A. J. \& Scott, P. 2009, ARA\&A, 47, 481

Bai, X.-N. \& Goodman, J. 2009, ApJ, 701, 737

Baulch, D. L. et al. 2005, J. Phys. Chem. Ref. Data, 34, 757

Bergin, E., Calvet, N., D’Alessio, P., \& Herczeg, G. J. 2003, ApJ, 591, L159

Bethell, T., \& Bergin, E. 2009, Science, 326, 1675

Carr, J. S. \& Najita, J. R. 2011, arXiv:1104.0184

Carr, J. S. \& Najita, J. R. 2008, Science, 319, 1504

Carr, J. S., Tokunaga, A. T., \& Najita, J. 2004, ApJ, 603, 213

Carmona, A. 2000, Earth Moon and Planets, 106, 71

Cazaux, S. \& Tielens 2002, A. G. G. M., ApJ, 575, L29

Cazaux, S. \& Tielens 2004, A. G. G. M., ApJ, 604, 222

Cazaux, S., Caselli, P., Tielens, A. G. G. M., Le Bourlet, J. \& Walmsley, M. 2005, J Phys, Conf. Series, 6, 155

Cazaux, S. \& Tielens, A. G. G. M. 2010, ApJ, 715, 608

Ciesla, F. J., \& Cuzzi, J. N. 2006, Icarus, 181, 178

Cuppen, H. M., Kristensen, L. E., \& Gavardi, E. 2010, MNRAS, 406, L11

D'Alessio, P., Calvet, N., Hartmann, L., Lizano, S., \& Cantó, J. 1999, ApJ, 527, 893

Doppmann, G. W., Najita, J. R., Carr, J. S., \& Graham, J. R. 2011, ApJ, 738, 112

Feigelson et al. 2005, ApJS, 160, 379

Furlan, E., et al. 2006, ApJS, 165, 568

Glassgold, A. E., Meijerink, R. \& Najita, J. 2009, ApJ, 701, 142 (GMN09)

Glassgold, A. E., \& Najita, J. 2001, in ASP Conf. Ser. 244, Young Stars near Earth: Progress and Prospects, ed. R. Jayawardhana \& T. P. Greene (San Francisco: ASP), 251

Glassgold, A. E., Najita, J. \& Igea, J. 2004, ApJ, 615, 972 (GNI04)

Gorti, U. \& Hollenbach, D. 2008, ApJ, 683, 287

Heinzeller, D., Nomura, H., Walsh, C., \& Millar, T. J. 2011, ApJ, 731, 115 
Hirose, S. \& Turner, N. J. 2011, ApJ, 732, 30

Jenkins, E. B. 2009, ApJ, 700, 1200

Ilgner, M., Henning, Th., Markwick, A. J.,\& Millar, T. J. 2004, A\&A, 415, 643

Kamp, I., \& Dullemond, C. P. 2004, ApJ, 615, 991

Kamp, I., Freudling, W., \& Chengalur, J. N. 2007, ApJ, 660,469

Kress, M. E., Tielens, A. G. G. M., \& Frenklach, M. 2010, Adv. Sp. Res., 46, 44

Markwick, A. J., Ilgner, M., Millar, T. J., Henning, Th. 2002, A\&A, 385, 632

Meijerink, R., Pontoppidan, K. M., Blake, G. A., Poelman, D. R., \& Dullemond, C. P. 2009, ApJ, 704, 1471

Najita, J. R., Carr, J. S., Glassgold, A. E., \& Valenti, J. A. 2007, Protostars and Planets V, ed. B. Reipurth, D. Jewitt, \& K. Keil (Tucson: Univ. of Arizona), 507

Najita, J. R., Carr, J. S., \& Mathieu, R. D. 2003, ApJ, 589,931

Najita, J. R., Doppmann, G. W., Carr, J. S., Graham, J. R., \& Eisner, J. A. 2009, ApJ, 691, 738

Najita, J. R., et al. 2010, ApJ, 712, 274

Nomura, H., Aikawa, Y., Tsujimoto, M., Nakagawa, Y., \& Millar, T. J. 2007, ApJ, 661, 334

Nomura, H., Aikawa, Y., Nakagawa, Y., \& Millar, T. J. 2009, AA, 495, 183

Nomura, H., \& Millar, T. J. 2005, A\&A, 438, 923

Pascucci, I., Apai, D., Luhman, K., Henning, Th., Bouwman, J., Meyer, M. R., Lahuis, F., \& Natta, A. 2009, ApJ, 696, 143

Parkinson, W. H. \& Yoshino, K. 2003, Chem. Phys., 294, 31

Pontoppidan, K. M., Salyk C., Blake, G. A., Meijerink, R., Carr, J. S., \& Najita, J. 2010, ApJ, 720, 887

Preibisch, T. \& Feigelson, E. D. 2005, ApJS, 160, 390

Roberge, W., Jones, D., Lepp, S., \& Dalgarno, A. 1991, ApJS, 77, 287

Salyk, C., Pontoppidan, K. M., Blake, G. A., Najita, J. R., \& Carr, J. S. 2011, ApJ, 731, 130

Salyk, C., Pontoppidan, K. M., Blake, G. A., Lahuis, F., van Dishoeck, E. F., \& Evans, N. J. II 2008, ApJ, 676, L49

Savage, B. D. \& Sembach, K. R. 1996, ARA\&A, 34, 279

Semenov, D., Wiebe, D., \& Henning, Th. 2006, ApJ, 647, L57

Teske, J. K., Najita, J. R., Carr, J. S., Pascucci, I., Apai, D., \& Henning, T. 2011, arXiv:1104.0249

Wakelam, V. 2009, BAAS, 41, 665 http://kida.obs.u-bordeaux1.fr

Walsh, C., Millar, 'T. J., \& Nomura, H. 2010, ApJ, 722, 1607

Woitke, P., Kamp, I., \& Thi, W.-F. 2009, A\&A, 501, 383

Willacy, K., Langer, W., Allen, M., \& Bryden, G. 2006, ApJ, 644, 1202

Willacy, K. \& Woods, M. 2009, ApJ, 703, 479

Woodall, J., Agúndez, M., Markwick-Kemper, A. J. \& Millar, T. J. 2007, A\&A, 466, 1197 http://www.udfa.net 
Table 1. Elemental Abundances

\begin{tabular}{|ccc|}
\hline Element & Abundance & Depletion \\
\hline \hline $\mathrm{H}$ & 1.00 & 1.0 \\
$\mathrm{He}$ & 0.10 & 1.0 \\
$\mathrm{C}$ & $1.40 \times 10^{-4}$ & 2.0 \\
$\mathrm{~N}$ & $6.00 \times 10^{-5}$ & 1.1 \\
$\mathrm{O}$ & $3.50 \times 10^{-4}$ & 1.4 \\
$\mathrm{Ne}$ & $6.90 \times 10^{-5}$ & 1.2 \\
$\mathrm{Na}$ & $2.31 \times 10^{-7}$ & 7.5 \\
$\mathrm{Mg}$ & $1.00 \times 10^{-6}$ & 40 \\
$\mathrm{~K}$ & $8.57 \times 10^{-9}$ & 12 \\
$\mathrm{Si}$ & $1.68 \times 10^{-6}$ & 19 \\
$\mathrm{~S}$ & $1.40 \times 10^{-5}$ & 1.0 \\
$\mathrm{Ar}$ & $1.51 \times 10^{-6}$ & 1.7 \\
$\mathrm{Fe}$ & $1.75 \times 10^{-7}$ & 180 \\
\hline
\end{tabular}

Table 3. Warm Columns (200-2000 K) ${ }^{\dagger}$

\begin{tabular}{|c|c|c|c|}
\hline$h$ & $1.0^{*}$ & 1.0 & 0.01 \\
\hline$a_{g}$ & $0.707^{*}$ & 7.07 & 0.707 \\
\hline$\overline{\mathrm{H}_{2}}$ & 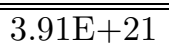 & 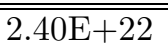 & $1.33 \mathrm{E}+21$ \\
\hline $\mathrm{H}^{+}$ & $3.20 \mathrm{E}+12$ & $3.60 \mathrm{E}+10$ & $9.56 \mathrm{E}+15$ \\
\hline $\mathrm{H}_{3}^{+}$ & $1.92 \mathrm{E}+13$ & $1.45 \mathrm{E}+12$ & $2.78 \mathrm{E}+13$ \\
\hline $\mathrm{He}^{+}$ & $7.75 \mathrm{E}+12$ & $5.00 \mathrm{E}+11$ & $2.28 \mathrm{E}+14$ \\
\hline $\mathrm{O}$ & $1.70 \mathrm{E}+17$ & $3.70 \mathrm{E}+16$ & $1.76 \mathrm{E}+17$ \\
\hline $\mathrm{OH}$ & $4.57 \mathrm{E}+14$ & $6.83 \mathrm{E}+13$ & $1.31 \mathrm{E}+15$ \\
\hline $\mathrm{H}_{2} \mathrm{O}$ & $40 \mathrm{E}+17$ & $5.29 \mathrm{E}+18$ & $1.96 \mathrm{E}+17$ \\
\hline $\mathrm{H}_{3} \mathrm{O}^{+}$ & $4.22 \mathrm{E}+13$ & $1.36 \mathrm{E}+13$ & $7.50 \mathrm{E}+13$ \\
\hline $\mathrm{O}_{2}$ & $3.72 \mathrm{E}+17$ & $1.73 \mathrm{E}+18$ & $1.05 \mathrm{E}+17$ \\
\hline $\mathrm{CO}$ & 8 & $6.55 \mathrm{E}$ & $4.30 \mathrm{E}+17$ \\
\hline $\mathrm{CO}_{2}$ & 15 & $1.10 \mathrm{E}$ & $2.25 \mathrm{E}+14$ \\
\hline $\mathrm{HCO}$ & 16 & $8.53 \mathrm{H}$ & $3.54 \mathrm{E}+14$ \\
\hline $\mathrm{HCC}$ & 13 & $1.49 \mathrm{E}+12$ & $4.60 \mathrm{E}+13$ \\
\hline $\mathrm{C}$ & $68 \mathrm{E}+14$ & $3.33 \mathrm{E}+13$ & $9.34 \mathrm{E}+15$ \\
\hline $\mathrm{CH}_{3}$ & $3.78 \mathrm{E}+09$ & $2.11 \mathrm{E}+10$ & $1.41 \mathrm{E}+09$ \\
\hline $\mathrm{CH}_{4}$ & $9.59 \mathrm{E}+11$ & $1.28 \mathrm{E}+14$ & $7.35 \mathrm{E}+10$ \\
\hline $\mathrm{C}_{2} \mathrm{H}_{2}$ & $1.12 \mathrm{E}+13$ & $5.53 \mathrm{E}+14$ & $1.36 \mathrm{E}+12$ \\
\hline $\mathrm{C}^{+}$ & $4.11 \mathrm{E}+12$ & $1.33 \mathrm{E}+11$ & $1.22 \mathrm{E}+15$ \\
\hline $\mathrm{CH}_{3}^{+}$ & $5.48 \mathrm{E}+11$ & $1.69 \mathrm{E}+11$ & $1.41 \mathrm{E}+11$ \\
\hline $\mathrm{C}_{2} \mathrm{H}_{3}^{+}$ & $3.79 \mathrm{E}+08$ & $3.08 \mathrm{E}+08$ & $3.73 \mathrm{E}+07$ \\
\hline $\mathrm{N}$ & $E+16$ & $1.24 \mathrm{E}+17$ & $4.59 \mathrm{E}+16$ \\
\hline $\mathrm{N}_{2}$ & $1.92 \mathrm{E}+17$ & $1.37 \mathrm{E}+18$ & $7.10 \mathrm{E}+16$ \\
\hline $\mathrm{NH}_{3}$ & -15 & 1.14 & $8.40 \mathrm{E}+14$ \\
\hline $\mathrm{HCN}$ & & 7.29 & $2.50 \mathrm{E}+14$ \\
\hline $\mathrm{NH}_{3}^{+}$ & 11 & $3.50 \mathrm{E}$ & $7.44 \mathrm{E}+11$ \\
\hline $\mathrm{N}_{2} \mathrm{H}$ & 12 & 1.42 & $2.73 \mathrm{E}+12$ \\
\hline $\mathrm{HCNH}$ & $1.96 \mathrm{E}+11$ & $3.67 \mathrm{E}+11$ & $3.20 \mathrm{E}+11$ \\
\hline NO & $7.29 \mathrm{E}+14$ & $1.23 \mathrm{E}+14$ & $1.55 \mathrm{E}+15$ \\
\hline$S$ & $1.28 \mathrm{E}+16$ & $8.65 \mathrm{E}+16$ & $1.42 \mathrm{E}+15$ \\
\hline $\mathrm{S}^{+}$ & $2.42 \mathrm{E}+13$ & $9.25 \mathrm{E}+11$ & $6.04 \mathrm{E}+14$ \\
\hline $\mathrm{SO}$ & $7.23 \mathrm{E}+14$ & $3.78 \mathrm{E}+15$ & $3.75 \mathrm{E}+14$ \\
\hline $\mathrm{SO}_{2}$ & $9.79 \mathrm{E}+16$ & $5.70 \mathrm{E}+17$ & $4.21 \mathrm{E}+16$ \\
\hline $\mathrm{CS}$ & $1.01 \mathrm{E}+14$ & $3.87 \mathrm{E}+14$ & $1.04 \mathrm{E}+13$ \\
\hline
\end{tabular}

${ }^{\dagger} a_{g}$ in $\mu \mathrm{m}$ and warm columns in $\mathrm{cm}^{-2}$.

* Reference case.

Table 2. Model Parameters

\begin{tabular}{|cccc|}
\hline Parameter & Symbol & Ref Value & Variation Range \\
\hline \hline Stellar Mass & $M_{*}$ & $0.5 M_{\odot}$ & \\
Stellar Radius & $R_{*}$ & $2 R_{\odot}$ & \\
Stellar Temperature & $T_{*}$ & $4000 \mathrm{~K}$ & \\
Disk Mass & $M_{D}$ & $0.005 M_{\odot}$ & \\
Disk Accretion Rate & $\dot{M}$ & $10^{-8} M_{\odot} \mathrm{yr}^{-1}$ & \\
Grain Size & $a_{g}$ & $0.707 \mu \mathrm{m}$ & $0.707-7.07 \mu \mathrm{m}$ \\
X-ray Luminosity & $L_{\mathrm{X}}$ & $2 \times 10^{30} \mathrm{erg} \mathrm{s}^{-1}$ & $10^{26}-10^{31} \mathrm{erg} \mathrm{s}^{-1}$ \\
X-ray Temperature & $T_{\mathrm{X}}$ & $1 \mathrm{keV}$ & $1-5 \mathrm{keV}$ \\
Mechanical Heating & $\alpha_{h}$ & 1.0 & $0.01-1.0$ \\
O/C Abundance Ratio & $\mathrm{O} / \mathrm{C}$ & 2.5 & $0.25-5$ \\
Radial Distance & $r$ & $1 \mathrm{AU}$ & $0.25-40 \mathrm{AU}$ \\
\hline
\end{tabular}


Table 4. Dependence of Warm Columns (200-2000 K) on $L_{\mathrm{x}}^{\dagger}$

\begin{tabular}{|c|c|c|c|c|c|c|c|}
\hline$L_{\mathrm{X}}$ & $.0 \mathrm{e} 26$ & $1.0 \mathrm{e} 27$ & $0 \mathrm{e} 28$ & $1.0 \mathrm{e} 29$ & $1.0 \mathrm{e} 30$ & $0 \mathrm{e} 30^{*}$ & , \\
\hline 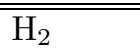 & $E$ & $84 \mathrm{E}$ & $E$ & $9 \mathrm{E}+21$ & $7 \mathrm{E}+21$ & +21 & 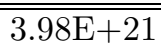 \\
\hline $\mathrm{H}^{+}$ & & & & & & & \\
\hline $\mathrm{H}_{3}^{+}$ & +09 & & & & & & \\
\hline $\mathrm{He}^{+}$ & $\mathrm{E}+08$ & $\mathrm{E}+09$ & $8 \mathrm{E}+10$ & $3 \mathrm{E}+11$ & $0 \mathrm{E}+12$ & +12 & $4 \mathrm{E}+1$ \\
\hline $\mathrm{O}$ & $\mathrm{E}+13$ & $5 \mathrm{E}+14$ & $1 \mathrm{E}+15$ & $\mathrm{E}+16$ & $7 \mathrm{E}+17$ & & $\mathrm{E}+1$ \\
\hline $\mathrm{OH}$ & $\mathrm{E}+11$ & $09 \mathrm{E}+12$ & $44 \mathrm{E}+13$ & $63 \mathrm{E}+13$ & $57 \mathrm{E}+14$ & & $\mathrm{E}+15$ \\
\hline $\mathrm{H}_{2} \mathrm{O}$ & $\mathrm{E}+17$ & $50 \mathrm{E}+17$ & $67 \mathrm{E}+17$ & $43 \mathrm{E}+17$ & $42 \mathrm{E}+17$ & $5.34 \mathrm{E}+17$ & $\mathrm{E}+17$ \\
\hline $\mathrm{H}_{3} \mathrm{O}^{-}$ & $\mathrm{E}+10$ & $15 \mathrm{E}+11$ & $65 \mathrm{E}+12$ & +12 & & & +1 \\
\hline $\mathrm{O}_{2}$ & +17 & +17 & & & & & +1 \\
\hline & +18 & $05 \mathrm{E}+18$ & & & & & +1 \\
\hline $\mathrm{CO}_{2}$ & & & & & & & \\
\hline & & & & & & & \\
\hline & & & & & & & \\
\hline $\mathrm{C}$ & & & & & & & \\
\hline & & & & & & & +0 \\
\hline & & & & & & & +1 \\
\hline & & & & 3 & & & +1 \\
\hline$C$ & & & & & & & $\mathrm{E}+1$ \\
\hline & & & 0 & 0 & 11 & & +1 \\
\hline $\mathrm{C}_{2}$ & 5 & & 7 & & 88 & 08 & $6.73 \mathrm{E}+0$ \\
\hline $\mathrm{N}$ & $\mathrm{E}+15$ & $39 \mathrm{E}+15$ & $26 \mathrm{E}+16$ & 16 & $21 \mathrm{E}+16$ & $8.21 \mathrm{E}+16$ & $1.14 \mathrm{E}+17$ \\
\hline $1 \sqrt{2}$ & +17 & $21 \mathrm{E}+17$ & $22 \mathrm{E}+17$ & $09 \mathrm{E}+17$ & 17 & 17 & $1.95 \mathrm{E}+17$ \\
\hline & -16 & 15 & 971 & 5 & 15 & & $2 \mathrm{E}+15$ \\
\hline & & & 5 & 4 & & & $2 \mathrm{E}+1$ \\
\hline & & & & & & & \\
\hline & -07 & +09 & $1.65 \mathrm{E}$ & $1.36 \mathrm{E}+11$ & $7.97 \mathrm{E}+11$ & $1.30 \mathrm{E}$ & $3.72 \mathrm{E}+12$ \\
\hline $\mathrm{HC}$ & & & & & & & +11 \\
\hline$N$ & & & & & & & $8 E+15$ \\
\hline N & - & & & & & & $0 \mathrm{E}+16$ \\
\hline $\mathrm{S}^{-}$ & & & & & & & +1 \\
\hline & & & & & & & \\
\hline $\mathrm{SO}_{2}$ & & & & & & & $1.02 \mathrm{E}+1$ \\
\hline$C C$ & 14 & 13 & 3 & 13 & $8.39 \mathrm{E}+$ & $9.64 \mathrm{E}+$ & $1.51 \mathrm{E}+1$ \\
\hline
\end{tabular}

${ }^{\dagger} L_{\mathrm{X}}$ in $\mathrm{erg} \mathrm{s}^{-1}$ and warm columns in $\mathrm{cm}^{-2}$.

* Reference case. 
Table 5. Dependence of Warm Columns $(200-2000 \mathrm{~K})$ on O/C Ratio ${ }^{\dagger}$

\begin{tabular}{|c|c|c|c|c|c|c|c|}
\hline $\mathrm{O} / \mathrm{C}$ & 0.25 & 0.50 & 1.00 & 1.50 & 2.00 & $2.5^{*}$ & 5.00 \\
\hline $\mathrm{H}_{2}$ & $\overline{85 \mathrm{E}+21}$ & $3.88 \mathrm{E}+21$ & $8 \overline{88 \mathrm{E}+21}$ & $3.91 \mathrm{E}+21$ & $3.91 \mathrm{E}+21$ & $3.91 \mathrm{E}+21$ & $3 \mathrm{E}+$ \\
\hline $\mathrm{H}^{+}$ & 3 & 4 & & 3 & 12 & $0 \mathrm{E}+12$ & 1 \\
\hline $\mathrm{H}_{3}^{+}$ & +13 & $06 \mathrm{E}+13$ & +13 & $E+13$ & +13 & +13 & +1 \\
\hline $\mathrm{He}^{+}$ & $3 \mathrm{E}+13$ & $95 \mathrm{E}+13$ & $42 \mathrm{E}+13$ & $05 \mathrm{E}+13$ & $81 \mathrm{E}+12$ & $75 \mathrm{E}+12$ & $4 \mathrm{E}+12$ \\
\hline $\mathrm{O}$ & $51 \mathrm{E}+12$ & $4.85 \mathrm{E}+12$ & $7 \mathrm{E}+17$ & $66 \mathrm{E}+17$ & $76 \mathrm{E}+17$ & $1.70 \mathrm{E}+17$ & $45 \mathrm{E}+17$ \\
\hline $\mathrm{OH}$ & $78 \mathrm{E}+11$ & $8.09 \mathrm{E}+11$ & $31 \mathrm{E}+13$ & $2.67 \mathrm{E}+14$ & $3.93 \mathrm{E}+14$ & $4.57 \mathrm{E}+14$ & $6.82 \mathrm{E}+14$ \\
\hline $\mathrm{H}_{2} \mathrm{O}$ & $68 \mathrm{E}+10$ & $1.64 \mathrm{E}+11$ & $15 \mathrm{E}+14$ & $1.48 \mathrm{E}+17$ & $3.45 \mathrm{E}+17$ & $5.40 \mathrm{E}+17$ & $1.62 \mathrm{E}+18$ \\
\hline $\mathrm{H}_{3} \mathrm{O}^{+}$ & $26 \mathrm{E}+08$ & $6.45 \mathrm{E}+08$ & $2.28 \mathrm{E}+12$ & $.78 \mathrm{E}+13$ & $3.68 \mathrm{E}+13$ & $4.22 \mathrm{E}+13$ & $6.24 \mathrm{E}+13$ \\
\hline $\mathrm{O}_{2}$ & $21 \mathrm{E}+08$ & $1.50 \mathrm{E}+09$ & $5.74 \mathrm{E}+13$ & $2.42 \mathrm{E}+16$ & $1.95 \mathrm{E}+17$ & $3.72 \mathrm{E}+17$ & $1.25 \mathrm{E}+18$ \\
\hline $\mathrm{CO}$ & $79 \mathrm{E}+16$ & $69 \mathrm{E}+16$ & $8.78 \mathrm{E}+17$ & $1.07 \mathrm{E}+18$ & $1.08 \mathrm{E}+18$ & $1.09 \mathrm{E}+18$ & $1.11 \mathrm{E}+18$ \\
\hline $\mathrm{CO}_{2}$ & $5 \mathrm{E}+12$ & $1.60 \mathrm{E}+13$ & $5.85 \mathrm{E}+14$ & $1.27 \mathrm{E}+15$ & $1.27 \mathrm{E}+15$ & $1.26 \mathrm{E}+15$ & $1.20 \mathrm{E}+15$ \\
\hline $\mathrm{HCO}$ & $64 \mathrm{E}+17$ & $5.12 \mathrm{E}+17$ & $91 \mathrm{E}+16$ & $17 \mathrm{E}+16$ & $.94 \mathrm{E}+16$ & $1.33 \mathrm{E}+16$ & $3.70 \mathrm{E}+15$ \\
\hline $\mathrm{HCC}$ & +12 & & & $\mathrm{E}+13$ & $\mathrm{E}+13$ & -13 & +13 \\
\hline $\mathrm{C}$ & & & & & & & \\
\hline $\mathrm{CH}_{3}$ & & & & 09 & & & \\
\hline & & & & & & & -11 \\
\hline C & 7 & & & 3 & & 13 & +12 \\
\hline $\mathrm{C}^{+}$ & $80 \mathrm{E}+13$ & 3 & 4 & 3 & 7.1 & 12 & +12 \\
\hline $\mathrm{CH}$ & $53 \mathrm{H}$ & $2.33 \mathrm{I}$ & 12 & $.03 \mathrm{E}-$ & $6.84 \mathrm{E}+11$ & $5.48 \mathrm{E}+11$ & $2.91 \mathrm{E}+11$ \\
\hline $\mathrm{C}_{2} \mathrm{H}_{3}^{+}$ & $51 \mathrm{E}+12$ & $8.55 \mathrm{E}+11$ & $77 \mathrm{E}+10$ & $1.53 \mathrm{E}+09$ & $6.80 \mathrm{E}+08$ & $3.79 \mathrm{E}+08$ & $5.23 \mathrm{E}+07$ \\
\hline $\mathrm{N}$ & $37 \mathrm{E}+17$ & $3.76 \mathrm{E}+17$ & 191 & $.57 \mathrm{E}+17$ & $1.10 \mathrm{E}+17$ & $9.01 \mathrm{E}+16$ & $5.34 \mathrm{E}+16$ \\
\hline $\mathrm{N}_{2}$ & $4.90 \mathrm{E}+16$ & $3.48 \mathrm{E}+16$ & +16 & $.59 \mathrm{E}+17$ & $1.82 \mathrm{E}+17$ & $1.92 \mathrm{E}+17$ & $2.12 \mathrm{E}+17$ \\
\hline $\mathrm{NH}_{3}$ & $4.76 \mathrm{E}+15$ & $5.67 \mathrm{E}+15$ & 051 & $1.20 \mathrm{E}+15$ & $2.87 \mathrm{E}+15$ & $3.42 \mathrm{E}+15$ & $3.69 \mathrm{E}+15$ \\
\hline $\mathrm{HCN}$ & $3 \mathrm{E}+16$ & $9.55 \mathrm{E}$ & 5 & $2.10 \mathrm{E}-$ & $1.17 \mathrm{E}+15$ & $8.05 \mathrm{E}+14$ & $2.90 \mathrm{E}+14$ \\
\hline $\mathrm{NH}$ & $17 \mathrm{H}$ & $1.45]$ & 2 & 1.2 & 7.5 & 5.30 & $2.35 \mathrm{E}+11$ \\
\hline $\mathrm{N}_{2} \mathrm{H}^{+}$ & $4.65 \mathrm{E}+11$ & $3.87 \mathrm{E}+11$ & $4.65 \mathrm{E}+11$ & $2.05 \mathrm{E}+12$ & $1.81 \mathrm{E}+12$ & $1.53 \mathrm{E}+12$ & $9.69 \mathrm{E}+11$ \\
\hline $\mathrm{NH}_{3}^{+}$ & $1.17 \mathrm{E}+12$ & $1.45 \mathrm{E}+12$ & $1.96 \mathrm{E}+12$ & $1.28 \mathrm{E}+12$ & $7.56 \mathrm{E}+11$ & $5.30 \mathrm{E}+11$ & $2.35 \mathrm{E}+11$ \\
\hline $\mathrm{HCNH}$ & $1.08 \mathrm{E}+13$ & & & 4.4 & & -11 & $1.23 \mathrm{E}+11$ \\
\hline $\mathrm{NO}$ & & & & & & 7.2 & +15 \\
\hline $\mathrm{S}$ & & & & & & & +16 \\
\hline $\mathrm{S}^{+}$ & & & & & & 2.4 & +13 \\
\hline $\mathrm{SO}$ & & & & & & -14 & +15 \\
\hline $\mathrm{SC}$ & -07 & $3.96 \mathrm{E}$ & 1. & $9.59 \mathrm{E}+16$ & $9.72 \mathrm{E}+16$ & $9.79 \mathrm{E}+16$ & $9.94 \mathrm{E}+16$ \\
\hline CS & $.55 \mathrm{E}+15$ & $1.44 \mathrm{E}+15$ & $1.55 \mathrm{E}+14$ & $9.78 \mathrm{E}+13$ & $1.07 \mathrm{E}+14$ & $1.01 \mathrm{E}+14$ & $5.20 \mathrm{E}+13$ \\
\hline
\end{tabular}

$\dagger$ Warm columns in $\mathrm{cm}^{-2}$.

* Reference case. 
Table 6. Dependence of Warm Columns (200-2000K) on Disk Radius ${ }^{\dagger}$

\begin{tabular}{|c|c|c|c|c|c|c|}
\hline $\mathrm{R}(\mathrm{AU})$ & 0.25 & 0.5 & $1^{*}$ & 2 & 4 & 10 \\
\hline$\overline{\overline{\mathrm{H}_{2}}}$ & $8 \overline{85+23}$ & $1.54 \mathrm{E}$ & $3.91 E+21$ & $\overline{35 \mathrm{E}+21}$ & 1.6 & 7.7( \\
\hline $\mathrm{H}^{+}$ & & $.81 \mathrm{E}+12$ & & & & +12 \\
\hline $\mathrm{H}_{3}^{+}$ & 3 & & & & & +12 \\
\hline $\mathrm{O}$ & $\mathrm{E}+16$ & $45 \mathrm{E}+17$ & +17 & 16 & & +16 \\
\hline $\mathrm{OH}$ & +14 & +14 & 14 & 14 & & +14 \\
\hline $\mathrm{H}_{2} \mathrm{O}$ & $\mathrm{E}+20$ & $75 \mathrm{E}+17$ & $40 \mathrm{E}+17$ & $17 \mathrm{E}+17$ & 3.00 & 5.40 \\
\hline $\mathrm{H}_{3} \mathrm{O}^{+}$ & $30 \mathrm{E}+14$ & $.21 \mathrm{E}+13$ & $4.22 \mathrm{E}+13$ & $.20 \mathrm{E}+13$ & 2.26 & $6.92 \mathrm{E}+12$ \\
\hline $\mathrm{O}_{2}$ & $54 \mathrm{E}+18$ & $2.08 \mathrm{E}+18$ & $.72 \mathrm{E}+17$ & $.85 \mathrm{E}+17$ & $1.28 \mathrm{E}+17$ & $7.81 \mathrm{E}+16$ \\
\hline $\mathrm{CO}$ & $30 \mathrm{E}+20$ & $4.28 \mathrm{E}+18$ & $1.09 \mathrm{E}+18$ & $.56 \mathrm{E}+17$ & +17 & $2.25 \mathrm{E}+17$ \\
\hline $\mathrm{CO}_{2}$ & & .77 & & & & \\
\hline & & 6 & & & & -11 \\
\hline $\mathrm{H}$ & & & & & & \\
\hline C & & & & & & 3.77 \\
\hline $\mathrm{CH}_{3}$ & & & & & & \\
\hline $\mathrm{CH}_{4}$ & & & & & & \\
\hline & & & & & & \\
\hline $\mathrm{C}^{+}$ & & & & & & -12 \\
\hline $\mathrm{CH}_{3}^{+}$ & & 7.73 & 5.4 & 2 & & 1.56 \\
\hline $\mathrm{C}_{2} \mathrm{H}_{3}^{+}$ & 8 & 5 & 3.7 ? & & & 8.0 \\
\hline$\lambda$ & 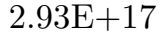 & 7 & & & & 1.5 \\
\hline $\mathrm{N}_{2}$ & 60 & 8.37 & 17 & 17 & 8.0 & $4.00 \mathrm{E}+16$ \\
\hline $\mathrm{NH}_{3}$ & $77 \mathrm{E}+18$ & $1.29 \mathrm{E}+16$ & $.42 \mathrm{H}$ & 1.76 & +15 & $6.51 \mathrm{E}+14$ \\
\hline $\mathrm{HCN}$ & $12 \mathrm{E}+18$ & $2.50 \mathrm{~F}$ & 14 & 4.991 & 3.5 & $1.34 \mathrm{E}+14$ \\
\hline $\mathrm{NH}_{3}^{+}$ & $22 \mathrm{E}+$ & 7.201 & .3 & 4.1 & 3.0 & $1.30 \mathrm{E}+11$ \\
\hline $\mathrm{N}_{2} \mathrm{H}$ & $89 \mathrm{E}-$ & $2.25 \mathrm{H}$ & 1.53 & 1.13 & 9. & 12 \\
\hline $\mathrm{HCNH}$ & 2 & 9 & 1.96 & & & 10 \\
\hline NO & 5 & 5 & & & & 6.6 \\
\hline S & & & & & & +12 \\
\hline $\mathrm{S}^{+}$ & $99 \mathrm{E}+13$ & $3.32 \mathrm{E}+13$ & $2.42 \mathrm{E}+13$ & 2.1 & 1.9 & $2.59 \mathrm{E}+11$ \\
\hline SO & 15 & 14 & 7.23 & & & $1.39 \mathrm{E}+13$ \\
\hline $\mathrm{SO}_{2}$ & $1.36 \mathrm{E}+19$ & $4.15 \mathrm{E}+17$ & $9.79 \mathrm{E}$ & 5.7 & 4.3 & $2.27 \mathrm{E}+16$ \\
\hline CC & $2.65 \mathrm{E}+14$ & $1.33 \mathrm{E}+14$ & $1.01 \mathrm{E}+14$ & $9.06 \mathrm{E}+13$ & $7.26 \mathrm{E}+13$ & $6.11 \mathrm{E}+09$ \\
\hline
\end{tabular}

$\dagger$ Warm columns in $\mathrm{cm}^{-2}$.

${ }^{*}$ Reference case. 


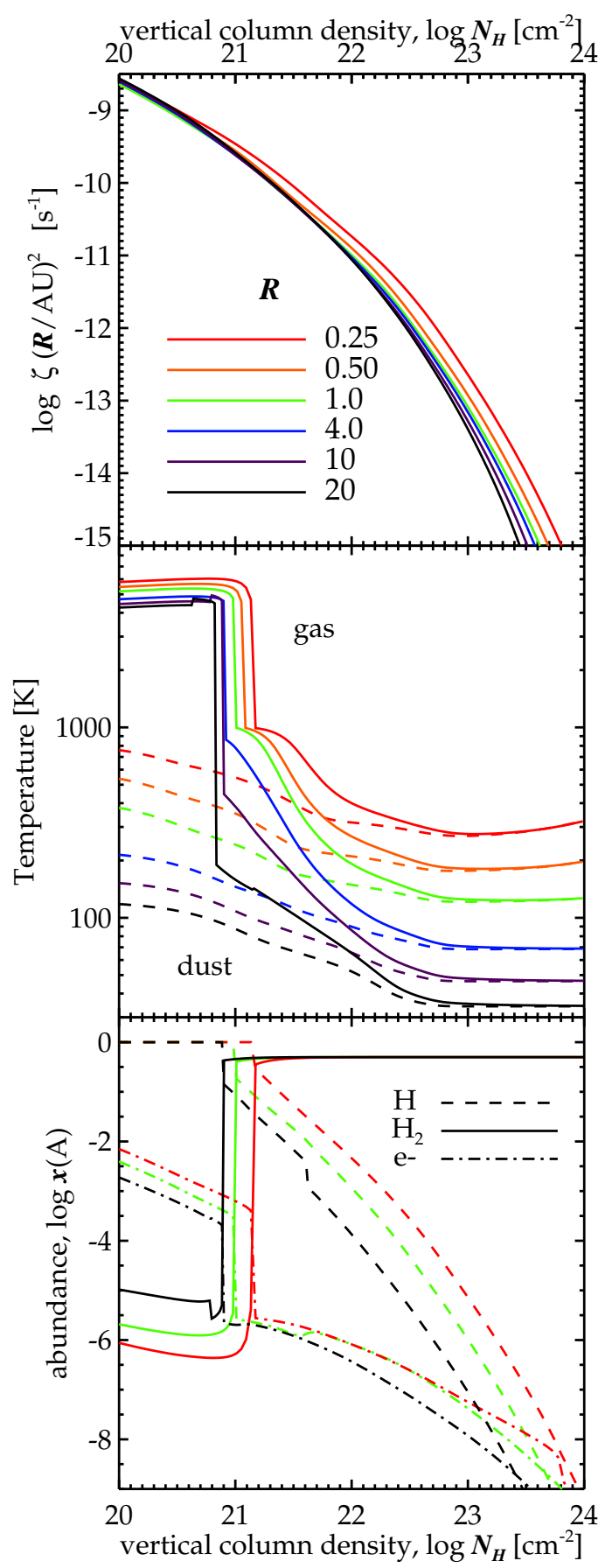

FIG. 1.- X-ray ionization rate (top panel), gas and dust temperature (middle panel), and abundances of atomic hydrogen, molecular hydrogen, and electrons (bottom panel) as a function of vertical column density and radius in the reference model. 


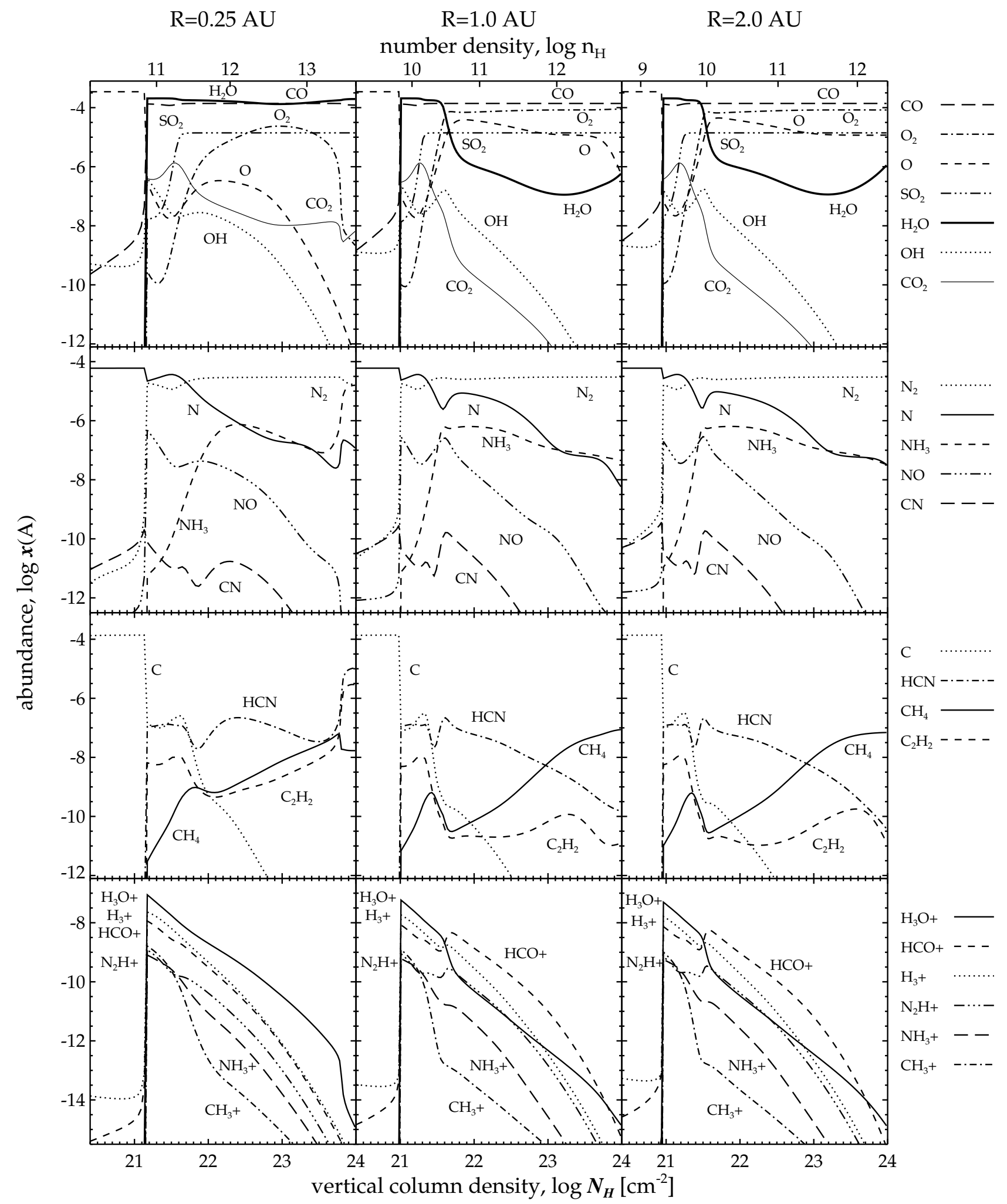

FIG. 2.- (a) Abundances of various species in the reference model at $0.25 \mathrm{AU}$ (left), $1 \mathrm{AU}$ (middle), and $2 \mathrm{AU}$ (right). 


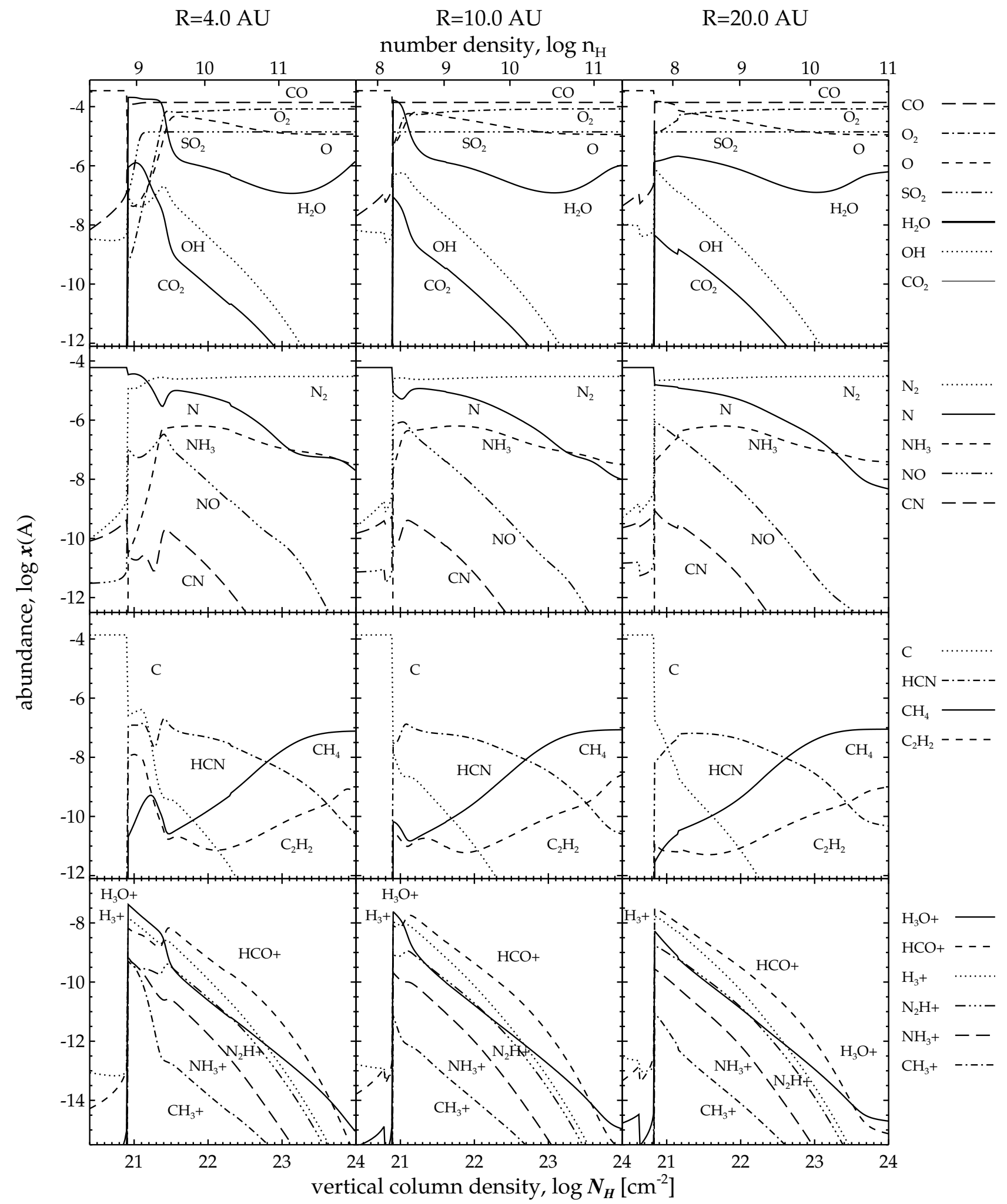

FIG. 2.- (b) Abundances of various species in the reference model at $4 \mathrm{AU}$ (left), $10 \mathrm{AU}$ (middle), and $20 \mathrm{AU}$ (right). 


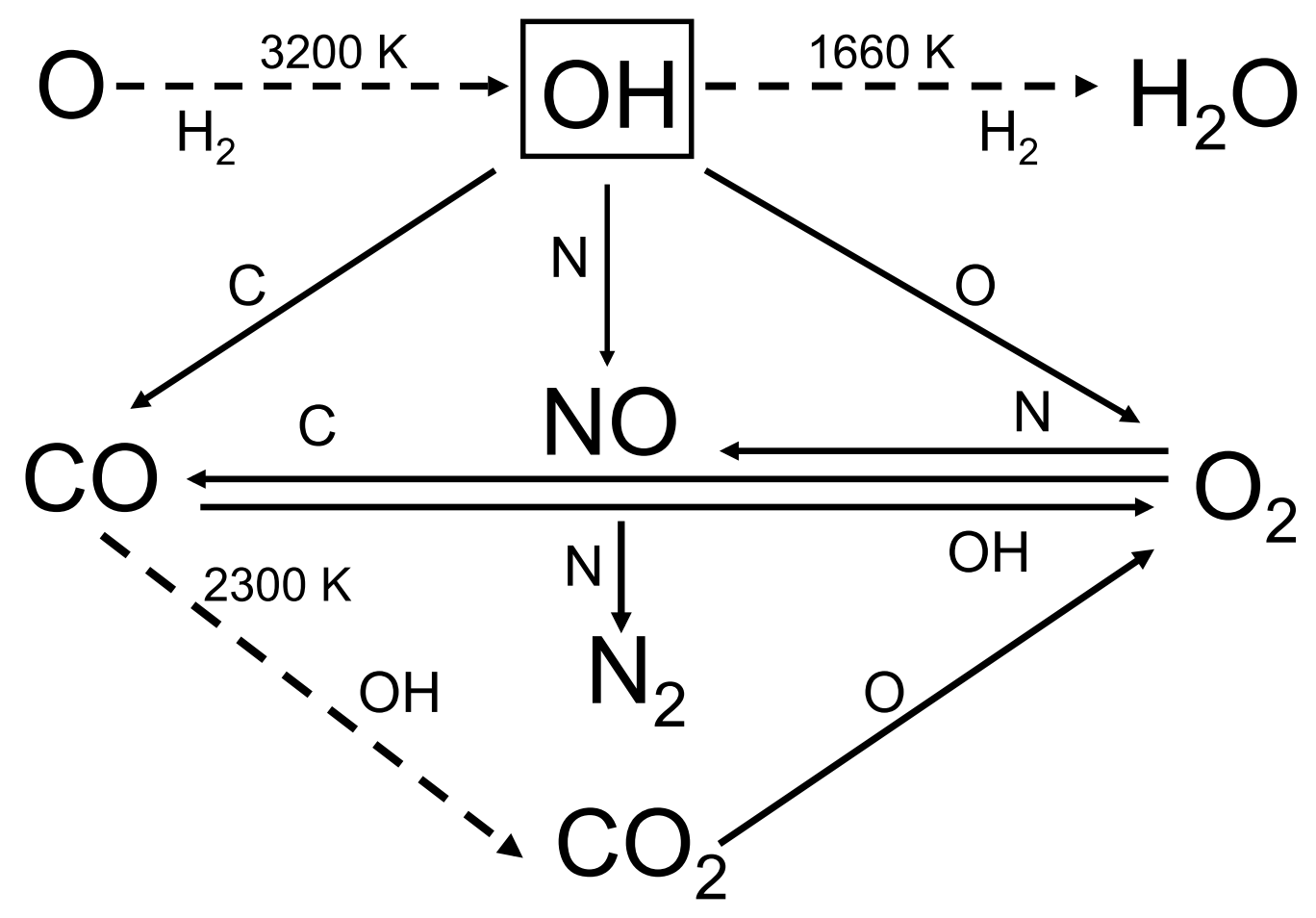

FIG. 3.- Schematic chemical network showing how fast neutral reactions of $\mathrm{OH}$ with $\mathrm{C}, \mathrm{O}$, and $\mathrm{N}$ atoms generate oxides that provide pathways to $\mathrm{CO}_{2}, \mathrm{O}_{2}$, and $\mathrm{N}_{2}$. 


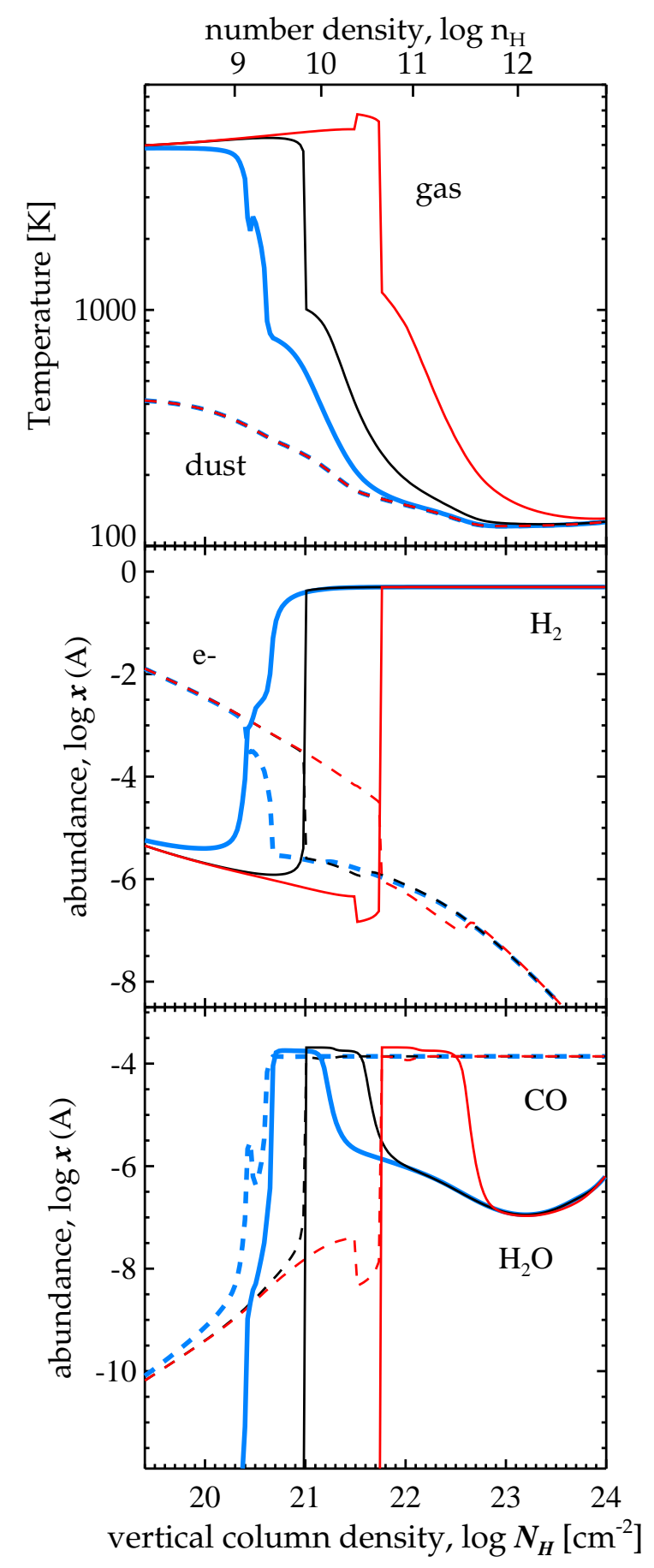

Fig. 4.- Gas (solid) and dust (dashed) temperatures (top panel) electron (dashed) and $\mathrm{H}_{2}$ (solid) abundances (middle panel), and $\mathrm{CO}$ (dashed) and water (solid) abundances (bottom panel) in the reference case (black), and for $\alpha_{h}=1, a_{g}=7.07$ (red) and $\alpha_{h}=$ $0.01, a_{g}=0.707$ (blue).

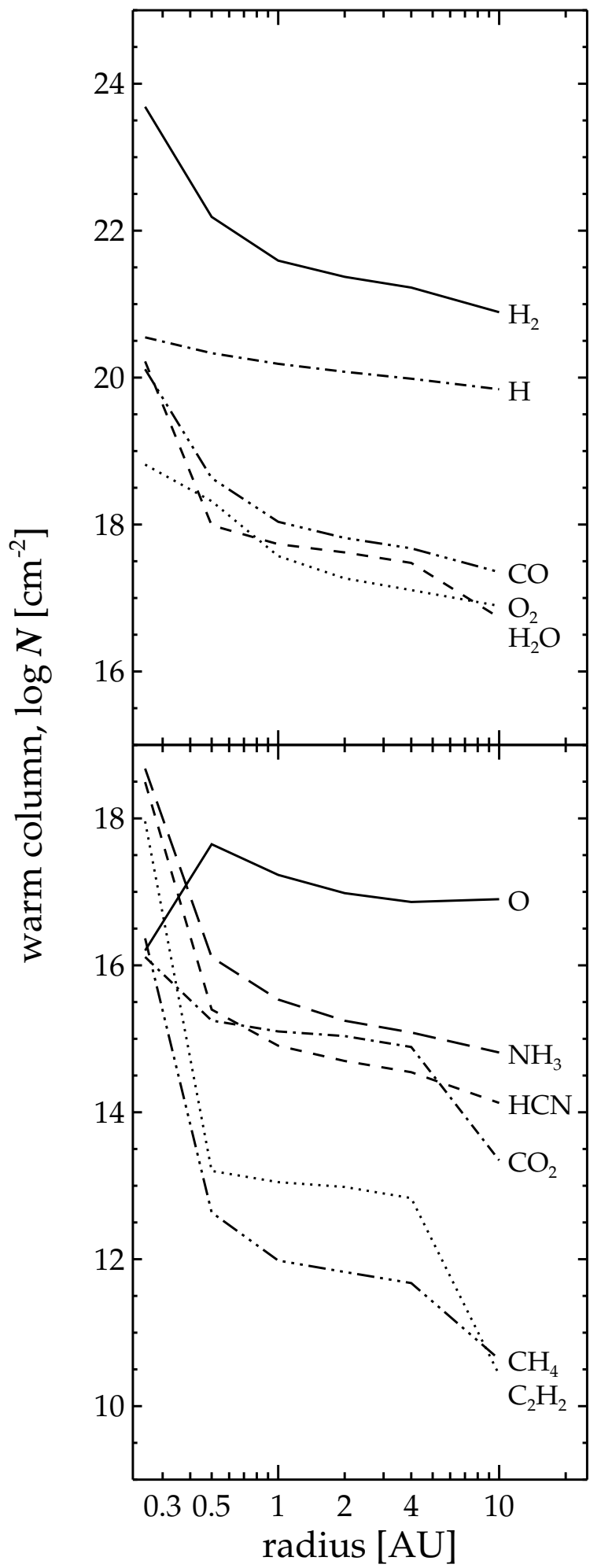

FIG. 5.- Warm columns of various species as a function of disk radius in the reference case. 